\title{
REAL ENTROPY RIGIDITY UNDER QUASI-CONFORMAL DEFORMATIONS
}

\author{
KHASHAYAR FILOM
}

\begin{abstract}
We set up a real entropy function $h_{\mathbb{R}}$ on the space $\mathcal{M}_{d}^{\prime}$ of Möbius conjugacy classes of real rational maps of degree $d$ by assigning to each class the real entropy of a representative $f \in \mathbb{R}(z)$; namely, the topological entropy of its restriction $f \uparrow_{\hat{\mathbb{R}}}$ to the real circle. We prove a rigidity result stating that $h_{\mathbb{R}}$ is locally constant on the subspace determined by real maps quasi-conformally conjugate to $f$. As examples of this result, we analyze real analytic stable families of hyperbolic and flexible Lattès maps with real coefficients along with numerous families of degree $d$ real maps of real entropy $\log (d)$. The latter discussion moreover entails a complete classification of maps of maximal real entropy.
\end{abstract}

\section{Contents}

1. Introduction

2. The real entropy function

3. Rigidity of real entropy

4. Examples of stable families of constant real entropy

References

\section{INTRODUCTION}

This article focuses on the entropy of real rational maps as a function defined on an appropriate moduli space. It is well known that a rational map $f: \hat{\mathbb{C}} \rightarrow \hat{\mathbb{C}}$ of degree $d \geq 2$ has topological entropy $\log (d)$ and admits a unique measure of maximal entropy $\mu_{f}$ whose support is the Julia set $\mathcal{J}(f)$ Lyu82, FLMn83, Mn83. In contrast, for $f \in \mathbb{R}(z)$, the topological entropy

$$
h_{\mathbb{R}}(f):=h_{\text {top }}\left(f \uparrow_{\hat{\mathbb{R}}}: \hat{\mathbb{R}} \rightarrow \hat{\mathbb{R}}\right)
$$

of the induced dynamics on the invariant circle $\hat{\mathbb{R}}:=\mathbb{R} \cup\{\infty\}$ can take any value between 0 and $\log (\operatorname{deg} f)$. In this paper, we study the real entropy $h_{\mathbb{R}}(f)$ both in relation with the dynamics of the ambient map $f: \hat{\mathbb{C}} \rightarrow \hat{\mathbb{C}}$ and also as $f$ varies in a family of real rational maps. Our main goal is to prove a rigidity statement showing that the real entropy is preserved in a family of quasi-conformally conjugate real maps. Let

$$
\mathcal{M}_{d}(\mathbb{C}):=\operatorname{Rat}_{d}(\mathbb{C}) / \mathrm{PGL}_{2}(\mathbb{C})
$$

Received by the editors May 4, 2019, and, in revised form, December 1, 2020.

2020 Mathematics Subject Classification. Primary 37B40, 37E10, 37F31; Secondary 37F10, $37 \mathrm{P} 45$. 
be the moduli space of one-dimensional holomorphic dynamical systems of degree $d$ where the group $\mathrm{PGL}_{2}(\mathbb{C})$ of Möbius transformations acts by conjugation on the space $\operatorname{Rat}_{d}(\mathbb{C})$ of rational maps of degree $d$. Consider the real subvariety

$$
\mathcal{M}_{d}^{\prime}:=\mathrm{PGL}_{2}(\mathbb{C}) \cdot\left(\operatorname{Rat}_{d}(\mathbb{R})\right) / \mathrm{PGL}_{2}(\mathbb{C})
$$

of dimension $2 d-2$ formed by classes with real representatives. The subvariety $\mathcal{M}_{d}^{\prime}$ is contained in the real locus $\mathcal{M}_{d}(\mathbb{R})$ of the moduli space. We have equality $\mathcal{M}_{d}^{\prime}=\mathcal{M}_{d}(\mathbb{R})$ if and only if $d$ is even; see Proposition 2.9.

Theorem 1.1. The real entropy (1.1) defines a continuous and surjective function

$$
h_{\mathbb{R}}: \mathcal{M}_{d}^{\prime}-\mathcal{S}^{\prime} \rightarrow[0, \log (d)]
$$

that for every $f \in \mathbb{R}(z)$ is constant on connected components of $\mathcal{M}_{d}^{\prime} \bigcap \mathrm{M}(f)-\mathcal{S}^{\prime}$.

Here, $\mathrm{M}(f)$ is the subspace of Möbius conjugacy classes of rational maps which are quasi-conformally conjugate to $f$ globally. The subspace $\mathcal{S}^{\prime}$ is the intersection with $\mathcal{M}_{d}^{\prime}$ of the symmetry locus $\mathcal{S}(\mathbb{C})$, which is the subset of conjugacy classes of degree $d$ maps that admit non-trivial Möbius automorphisms. In Proposition 2.4 we prove that $\mathcal{S}(\mathbb{C})$ is a closed subvariety of $\mathcal{M}_{d}(\mathbb{C})$ of dimension $d-1$; therefore, the domain $\mathcal{M}_{d}^{\prime}-\mathcal{S}^{\prime}$ of $h_{\mathbb{R}}$ is a real variety of dimension $2 d-2$. It is irreducible as a real variety but consists of $d+1$ connected components in its analytic topology; see Proposition 2.5. The continuity of $h_{\mathbb{R}}$ follows from Mis95.

The subvariety $\mathcal{S}^{\prime}=\mathcal{M}_{d}^{\prime} \cap \mathcal{S}(\mathbb{C})$ is excluded so that $h_{\mathbb{R}}$ is well defined: the dynamics on $\hat{\mathbb{R}}$ should be independent of the real representative picked from a Möbius conjugacy class so one has to omit real maps admitting twists; that is, real rational maps that are Möbius conjugate only over $\mathbb{C}$, and it is well known that twists are always associated with non-trivial automorphisms [Sil07, §4.8].

The dynamical moduli space $\mathrm{M}(f)$ associated with a rational map $f$ has been introduced and thoroughly studied in [MS98]. Any structurally-stable holomorphic family $\left\{f_{\lambda}\right\}_{\lambda \in \Lambda}$ of degree $d$ rational maps including $f$ comes with a canonical map $\Lambda \rightarrow \mathrm{M}(f)$ that to each parameter $\lambda$ assigns the conformal conjugacy class $\left\langle f_{\lambda}\right\rangle \in$ $\mathrm{M}(f) \subset \mathcal{M}_{d}(\mathbb{C})$. This is due to the fact that the notions of quasi-conformal and topological stability coincide [MS98, Theorem 7.1]. Hence the moduli spaces M $(f)$ provide a natural framework for Theorem 1.1 which is concerned with the constancy of the real entropy function $h_{\mathbb{R}}$ in a structurally-stable family of real rational maps. Nevertheless, as it is the Julia set that captures the relevant part of the dynamics of a rational map, it is not surprising that an analogous result can be formulated for families of real maps that are $\mathcal{J}$-stable in the sense of [MnSS83]:

Proposition 1.2. The real entropy is constant along any analytic 1-parameter $\mathcal{J}$-stable family $\left\{f_{t}\right\}_{t \in[0,1]}$ of real rational maps of degree $d$.

Hyperbolic components in $\mathcal{M}_{d}(\mathbb{C})$ are examples of $\mathcal{J}$-stable components of the moduli space. As for the entropy values over real hyperbolic components, the claim of Proposition 1.2 will be directly proved via invoking the kneading theory of Milnor and Thurston MT88 in 4.1. A non-hyperbolic but algebraic example of a structurally stable family contained in a single isentrope is the family of flexible Lattès maps for which the real entropy can be calculated explicitly; see $\$ 4.2$ There are also interesting families of quasi-conformally constant real rational maps where $h_{\mathbb{R}}(f)$ attains its maximum $\log (\operatorname{deg} f)$. Characterizing such rational maps turns out to be an interesting problem in its own right: 
Theorem 1.3. Let $f$ be a real rational map of degree $d \geq 2$ with $h_{\mathbb{R}}(f)=\log (d)$. Then the Julia set $\mathcal{J}(f)$ is completely contained in $\hat{\mathbb{R}}$ and either coincides with it or is a subinterval or a Cantor subset of it.

Moreover, in terms of Blaschke products

$$
\mathrm{e}^{2 \pi \mathrm{i} c} \prod_{i=1}^{d}\left(\frac{z-a_{i}}{1-\bar{a}_{i} z}\right) \quad\left(\left|a_{1}\right|, \ldots,\left|a_{d}\right|<1 ; c \in \mathbb{R} / \mathbb{Z}\right)
$$

that preserve the unit circle instead of the real circle $\hat{\mathbb{R}}$ :

(i) The map in (1.2) is hyperbolic with its Julia set a Cantor subset of the unit circle if and only if there is a $\theta_{0} \in \mathbb{R} / \mathbb{Z}$ satisfying

$$
\sum_{i=1}^{d} \frac{1-\left|a_{i}\right|^{2}}{\left|\mathrm{e}^{2 \pi \mathrm{i} \theta_{0}}-a_{i}\right|^{2}}<1
$$

for which

$$
c \equiv(d+1) \cdot \theta_{0}-\frac{1}{\pi} \sum_{i=1}^{d} \arg \left(\mathrm{e}^{2 \pi \mathrm{i} \theta_{0}}-a_{i}\right) \quad(\bmod \mathbb{Z}) .
$$

(ii) Up to conjugation with the Cayley transform $\left(z \mapsto \frac{z-\mathrm{i}}{z+\mathrm{i}}\right)$ and a biholomorphism of the unit disk, hyperbolic degree d maps $f$ with $\mathcal{J}(f)=\hat{\mathbb{R}}$ are the same as Blaschke products

$$
z \cdot \prod_{i=1}^{d-1}\left(\frac{z-a_{i}}{1-\bar{a}_{i} z}\right) \quad\left(\left|a_{1}\right|, \ldots,\left|a_{d-1}\right|<1\right)
$$

or their post-composition with $z \mapsto \frac{1}{z}$.

(iii) Every non-hyperbolic degree d map with $\mathcal{J}(f)=\hat{\mathbb{R}}$ after conjugation with a suitable real Möbius map would be a map of the form $\epsilon\left(z+\frac{P(z)}{Q(z)}\right)$ with non-real critical points where $\epsilon \in\{ \pm 1\}$ and $P, Q$ are coprime real polynomials satisfying $\operatorname{deg} P \leq d-1$ and $\operatorname{deg} Q=d-1$ with the inequality strict when $\epsilon=+1$.

(iv) Every degree $d$ map $f$ with the Julia set a subinterval of $\hat{\mathbb{R}}$ is semiconjugate to a map from one of the classes appeared in the last two parts: up to a real Möbius conjugacy, $f$ is either the quotient of a map such as

$$
\pm z \cdot \prod_{i=1}^{d-1}\left(\frac{z-a_{i}}{1-\overline{a_{i} z}}\right) \quad\left(\begin{array}{c}
\left|a_{1}\right|, \ldots,\left|a_{d-1}\right|<1 \\
\left\{a_{1}, \ldots, a_{d-1}\right\}=\left\{\overline{a_{1}}, \ldots, \overline{a_{d-1}}\right\}
\end{array}\right)
$$

by the action of $z \mapsto \frac{1}{z}$ that commutes with it; or is the quotient of a map of the form $\epsilon\left(z+\frac{P(z)}{Q(z)}\right)$ appeared in (iii) by the action of $z \mapsto-z$ provided that it commutes with $\epsilon\left(z+\frac{P(z)}{Q(z)}\right)$; namely, either $P(z)$ is odd and $Q(z)$ is even or vice versa.

Part (i) furnishes us with a $\mathcal{J}$-stable family of rational maps whose Julia sets are Cantor subsets of the unit (or equivalently real) circle while the other parts classify all rational maps of maximal real entropy whose Julia sets are one of the other alternatives, namely a real subinterval or the whole real circle. Rational maps with circle or interval Julia sets have also been studied in [EvS11] and [Fat19, §25]. 
Motivation. An important question regarding the real entropy function on the moduli space of real rational maps is the nature of its level sets, called isentropes. We view this article as a first step towards the study of the level sets of the function $h_{\mathbb{R}}$ defined on the moduli of real rational maps. There is an extensive literature on the monotonicity of entropy for various families of polynomials where the central problem is the connectedness of isentropes. The monotonicity was first established by Milnor and Thurston for the quadratic polynomial family (in [MT88]; also see [DH85, Dou95]), and for bimodal cubic polynomials by Milnor and Tresser in [MT00]. The general case of degree $d$ polynomials with $d$ real non-degenerate critical points was settled in BvS15] by van Strien and Bruin. The setting of rational maps is different from the polynomial setting because we are dealing with circle maps $f \uparrow_{\hat{\mathbb{R}}}: \hat{\mathbb{R}} \rightarrow \hat{\mathbb{R}}$ instead of interval maps: In all aforementioned references one essentially deals with a boundary-anchored map of an interval outside which the orbits either escape to infinity or converge to a cycle of period at most two [MT00, Theorem 3.2]. Of course, if the restriction is not surjective, then $f \uparrow_{\hat{\mathbb{R}}}: \hat{\mathbb{R}} \rightarrow \hat{\mathbb{R}}$ can be replaced with an (not necessarily boundary-anchored) interval map of the same entropy; as

$$
h_{\mathbb{R}}(f)=h_{\text {top }}\left(f \uparrow_{\hat{\mathbb{R}}}: \hat{\mathbb{R}} \rightarrow \hat{\mathbb{R}}\right)=h_{\text {top }}\left(f \uparrow_{f(\hat{\mathbb{R}})}: f(\hat{\mathbb{R}}) \rightarrow f(\hat{\mathbb{R}})\right) .
$$

For example, when the rational map $f$ is quadratic; $f \uparrow_{\hat{\mathbb{R}}}: \hat{\mathbb{R}} \rightarrow \hat{\mathbb{R}}$ is either a covering map (and thus of entropy $\log (2)$ according to MS80, Theorem $\left.1^{\prime}\right]$ ) or is not surjective in which case one can work with the interval map $f \uparrow_{f(\hat{\mathbb{R}})}: f(\hat{\mathbb{R}}) \rightarrow f(\hat{\mathbb{R}})$ instead; cf. [Mil93, §10]. It turns out that in general the domain $\mathcal{M}_{d}^{\prime}-\mathcal{S}^{\prime}$ of the real entropy function is disconnected (Proposition 2.5) and thus the monotonicity has to be studied among maps whose restrictions to $\hat{\mathbb{R}}$ are of a common topological degree. There are even smaller natural analytic domains in $\mathcal{M}_{d}^{\prime}$ that are dynamically defined according to the degree along with the modality of $f \uparrow_{f(\hat{\mathbb{R}})}$; and the monotonicity of the restriction of $h_{\mathbb{R}}$ to such regions is still worthy to investigate. As an example, for $d=2$ a natural partition of $\mathcal{M}_{2}^{\prime}=\mathcal{M}_{2}(\mathbb{R}) \cong \mathbb{R}^{2}$ to degree \pm 2 , monotonic, unimodal and bimodal regions has been outlined in [Mil93, §10]. The entropy behavior over $\mathcal{M}_{2}^{\prime}$ is the subject of the article [Fil19] that focuses on the monotonicity problem.

Outline. Putting the real entropy function $h_{\mathbb{R}}$ (as described in Theorem 1.1) on a firm footing is the main goal of second section and is done in $\$ \$ 2.12 .22 .3$ The last subsection of $₫ 2$ discusses how $\mathcal{M}_{d}^{\prime}$ is related to the real locus $\mathcal{M}_{d}(\mathbb{R})$.

The main result, Theorem 1.1 is formulated in the context of the theory of Teichmüller and moduli spaces of rational maps developed by McMullen and Sullivan in MS98. After a brief overview of this theory, we prove the theorem in 93 and discuss its implications for the dimension of isentropes; see Corollary 3.5

The fourth section is devoted to various examples of (structurally or $\mathcal{J}$-) stable families of rational maps with real coefficients that, according to our rigidity result, should come with the same real entropy. First, in 4.1 we directly prove that $h_{\mathbb{R}}$ is locally constant on the real locus of a hyperbolic component in $\mathcal{M}_{d}(\mathbb{C})$; see Theorem 4.1. In 4.2 we calculate the real entropy for families of flexible Lattès maps with real coefficients. Finally, in 44.3 we turn to the isentrope $h_{\mathbb{R}}=\log (d)$ where $h_{\mathbb{R}}$ : $\mathcal{M}_{d}^{\prime}-\mathcal{S}^{\prime} \rightarrow[0, \log (d)]$ attains its maximum. A careful analysis in Theorems 4.7 and 4.8 of the dynamics induced on the real circle by a map $f \in \operatorname{Rat}_{d}(\mathbb{R})$ with $h_{\mathbb{R}}(f)=\log (d)$ culminates in families of degree $d$ maps of real entropy $\log (d)$ 
outlined in Theorem 1.3 Each of these families is structurally stable for a generic choice of parameters and (once projected to $\mathcal{M}_{d}^{\prime}$ ) parametrizes the real locus of a single dynamical moduli space $\mathrm{M}(f)$ associated with a degree $d$ real map $f$ with $h_{\mathbb{R}}(f)=\log (d)$.

Notation and Terminology. A lap of an interval map $f: I \rightarrow I$ is defined to be a maximal monotonic subinterval and the number of laps (i.e. modality +1 ) is denoted by $l(f)$ and is called the lap number. It is a standard fact that the entropy of a multimodal (i.e. piecewise monotone or of finite modality) interval map is the exponential growth rate of the number of laps of its iterates [MS80, Theorem 1]:

$$
h_{\mathrm{top}}(f)=\lim _{n \rightarrow \infty} \frac{1}{n} \log \left(l\left(f^{\circ n}\right)\right)=\inf _{n} \frac{1}{n} \log \left(l\left(f^{\circ n}\right)\right) .
$$

The result remains valid for a multimodal self-map of the circle $S^{1}$ as long as "laps" are interpreted as those of the (possibly discontinuous) transformation of $[0,1$ ) obtained from conjugating the transformation of $S^{1}$ with $[0,1) \rightarrow S^{1}: x \mapsto \mathrm{e}^{2 \pi \mathrm{i} x}$ [MS80, Theorem $\left.1^{\prime}\right]$. In particular, the $n^{\text {th }}$ iterate of a degree $d$ covering $S^{1} \rightarrow S^{1}$ lifts to a self-map of $[0,1)$ with $d^{n}$ continuous pieces, all of them monotonic. Its entropy is thus $\log (d)$.

Spaces $\hat{\mathbb{C}}$ and $\hat{\mathbb{R}}$ denote the compactifications $\mathbb{C} \cup\{\infty\}$ and $\mathbb{R} \cup\{\infty\}$ of $\mathbb{C}$ and $\mathbb{R}$ respectively and we use $z$ and $x$ for the coordinates on them. When the Riemann sphere is considered as a complex algebraic curve, the notation $\mathbb{P}^{1}(\mathbb{C})$ is used instead. The degree of a rational map is denoted by $d$ and is always assumed to be greater than one. The Julia set of a rational map $f$ is shown by $\mathcal{J}(f)$.

For notations related to the moduli space of rational maps, we mainly follow Mil93 and Sil07: the moduli space of rational maps of degree $d$ is an affine variety $\mathcal{M}_{d}$ with a model over the rational numbers constructed by Silverman in Sil98 as the geometric quotient Rat ${ }_{d} / \mathrm{PSL}_{2}$ where Rat ${ }_{d} \subset \mathbb{P}^{2 d+1}$ is the parameter space of degree $d$ rational maps. Given a variety $V$ over a field $K$, the set of its $K^{\prime}$-points is written as $V\left(K^{\prime}\right)$ for any larger field $K^{\prime} \supseteq K$. So here it makes sense to write $\mathcal{M}_{d}(K)$ for any subfield $K$ of $\mathbb{C}$, and the complex variety $\mathcal{M}_{d}(\mathbb{C})$ coincides with the orbifold $\operatorname{Rat}_{d}(\mathbb{C}) / \mathrm{PGL}_{2}(\mathbb{C})$ consisting of Möbius conjugacy classes of degree $d$ rational maps on the Riemann sphere. The conjugacy class of a rational map $f$ will be denoted by $\langle f\rangle$. The subvariety $\mathcal{S}(\mathbb{C})$ of $\mathcal{M}_{d}(\mathbb{C})$, called the symmetry locus, is the subvariety determined by rational maps $f$ for which the group $\operatorname{Aut}(f)$ of Möbius transformations commuting with $f$ is non-trivial. The rational map $\bar{f}$ is the map obtained by applying the complex conjugation to coefficients of $f \in \mathbb{C}(z)$. For the broader class of complex-valued functions on $\hat{\mathbb{C}}$, we use the notation $\tilde{f}$ instead which is the result of conjugating $z \mapsto f(z)$ with $z \mapsto \bar{z}$; cf. (3.1). As for special transformations, the antipodal involution and the Joukowsky transform are given by $\gamma:=z \mapsto \frac{-1}{\bar{z}}$ and $\mathfrak{j}:=z \mapsto z+\frac{1}{z}$ respectively. Finally, in speaking of quasi-conformal (q.c. for short) homeomorphisms of the Riemann sphere, the dilatation is always denoted by $\mu$.

\section{The REAL ENTROpy FUnCtion}

The real entropy (1.1) can be considered as a well defined continuous function on a certain open submanifold of $\mathcal{M}_{d}^{\prime} \subset \mathcal{M}_{d}(\mathbb{C})$ obtained from omitting those $\mathrm{PGL}_{2}(\mathbb{C})$-conjugacy classes of real maps that include more than one $\mathrm{PGL}_{2}(\mathbb{R})$ conjugacy class. Studying this domain is the subject of the first two subsections 
$\S \$ 2.12 .2$ Properties of the real entropy function stated in Theorem 1.1 will be proved in Proposition 2.7 of $\$ 2.3$, In $\$ 2.4$ we address the natural question of how the real locus $\mathcal{M}_{d}(\mathbb{R})$ of $\mathcal{M}_{d}(\mathbb{C})$ is related to the locus $\mathcal{M}_{d}^{\prime}$ determined by rational maps that actually admit a model over $\mathbb{R}$; see Proposition 2.9

2.1. Symmetries. The real entropy is assigned to rational maps with real coefficients, so it is natural to form the subspace

$$
\mathcal{M}_{d}^{\prime}:=\mathrm{PGL}_{2}(\mathbb{C}) .\left(\operatorname{Rat}_{d}(\mathbb{R})\right) / \mathrm{PGL}_{2}(\mathbb{C})=\left\{\langle f\rangle \in \mathcal{M}_{d}(\mathbb{C}) \mid f \in \mathbb{R}(z)\right\}
$$

consisting of conjugacy classes in $\mathcal{M}_{d}(\mathbb{C})$ that contain a real map. The real entropy $h_{\mathbb{R}}$ is not well defined as a function on the entirety of this real subvariety because the ambiguity in picking a real representative can lead to different entropy values.

Example 2.1. Given $\mu \in \mathbb{R}-\{0\}$, real quadratic rational maps $\frac{1}{\mu}\left(z \pm \frac{1}{z}\right)$ are conjugate via $z \mapsto \mathrm{i} z$ but exhibit quite different dynamical behavior on the real circle. The critical points of $\frac{1}{\mu}\left(z-\frac{1}{z}\right)$ are not real, so it induces a degree two covering $x \mapsto \frac{1}{\mu}\left(x-\frac{1}{x}\right)$ of $\hat{\mathbb{R}}$ whose entropy is therefore $\log (2)$. On the other hand, the topological entropy of $x \mapsto \frac{1}{\mu}\left(x+\frac{1}{x}\right)$ vanishes: for $|\mu| \leq 1$ every orbit is attracted to the fixed point $\infty$ of multiplier $\mu$; for $\mu>1$ orbits in the invariant interval $(0, \infty)$ tend to the attracting fixed point $\frac{1}{\sqrt{\mu-1}}$ while those in the invariant interval $(-\infty, 0)$ tend to the attracting fixed point $-\frac{1}{\sqrt{\mu-1}}$; and finally, for $\mu<-1$ there is no finite real fixed point and any point of $\hat{\mathbb{R}}$, other than the fixed point $\infty$ and its preimage 0 , converges under iteration to the 2-cycle consisting of $\pm \frac{1}{\sqrt{-\mu-1}}$ whose multiplier is $\left(\frac{2+\mu}{\mu}\right)^{2}<1$.

The problem with the pair $\frac{1}{\mu}\left(z \pm \frac{1}{z}\right)$ from the preceding example is that they are conjugate over the complex numbers but not over the reals. We are interested in the dynamics of $f \uparrow_{\hat{\mathbb{R}}}$ and that is invariant only under real conjugacies, i.e. elements of $\mathrm{PGL}_{2}(\mathbb{R})$. In the literature of arithmetic dynamics, such examples of rational maps over a field $K$ which are conjugate only over a strictly larger field $K^{\prime}$ are called twists and they happen only if maps admit symmetries in $\mathrm{PGL}_{2}(\bar{K})$; see Sil07, $\S \S 4.7,4.8,4.9]$ for details. In our context, only twists over $\mathbb{R}$ are relevant.

Proposition 2.2. Fix $f \in \mathbb{R}(z)$ with $\operatorname{Aut}(f)=\{\mathbf{1}\}$. If $g \in \mathbb{R}(z)$ is $\mathrm{PGL}_{2}(\mathbb{C})$ conjugate to $f$, then $f, g$ are in fact $\mathrm{PGL}_{2}(\mathbb{R})$-conjugate.

Proof. Assume the contrary; the complex conjugacy class $\langle f\rangle$ contains at least two distinct real conjugacy classes. This implies the existence of a Möbius transformation $\alpha \in \mathrm{PGL}_{2}(\mathbb{C})-\mathrm{PGL}_{2}(\mathbb{R})$ for which $\alpha \circ f \circ \alpha^{-1} \in \mathbb{R}(z)$. But then taking complex conjugates implies that

$$
\bar{\alpha} \circ f \circ \overline{\alpha^{-1}}=\alpha \circ f \circ \alpha^{-1} \Rightarrow\left(\alpha^{-1} \circ \bar{\alpha}\right) \circ f \circ\left(\alpha^{-1} \circ \bar{\alpha}\right)^{-1}=f .
$$

Hence the non-identity Möbius map $\alpha^{-1} \circ \bar{\alpha}$ lies in Aut $(f)$; a contradiction.

Thus, in order to have a well defined real entropy function, we should remove from the space $\mathcal{M}_{d}^{\prime}$ in (2.1) the subspace of complex conjugacy classes of real maps with non-trivial Möbius symmetries; the subspace which will be denoted by

$$
\mathcal{S}^{\prime}:=\left\{\langle f\rangle \in \mathcal{M}_{d}(\mathbb{C}) \mid f \in \operatorname{Rat}_{d}(\mathbb{R}), \operatorname{Aut}(f) \neq\{\mathbf{1}\}\right\}
$$


herein. This is obviously the intersection of the complex symmetry locus

$$
\mathcal{S}(\mathbb{C})=\left\{\langle f\rangle \in \mathcal{M}_{d}(\mathbb{C}) \mid f \in \operatorname{Rat}_{d}(\mathbb{C}), \operatorname{Aut}(f) \neq\{\mathbf{1}\}\right\}
$$

with $\mathcal{M}_{d}^{\prime}$. So we arrive at a well defined entropy function:

Definition 2.3. For any $d \geq 2$, the real entropy function is defined as

$$
\left\{\begin{array}{l}
h_{\mathbb{R}}: \mathcal{M}_{d}^{\prime}-\mathcal{S}^{\prime} \rightarrow[0, \log (d)] \\
\langle f\rangle \mapsto h_{\text {top }}\left(f \uparrow_{\hat{\mathbb{R}}}: \hat{\mathbb{R}} \rightarrow \hat{\mathbb{R}}\right)
\end{array} \quad(f \in \mathbb{R}(z)) .\right.
$$

The domain of definition is $\left\{\langle f\rangle \in \mathcal{M}_{d}(\mathbb{C}) \mid f \in \mathbb{R}(z)\right.$, Aut $\left.(f)=\{\mathbf{1}\}\right\}$ and the codomain is $[0, \log (d)]$ since

$$
h_{\mathbb{R}}(f)=h_{\text {top }}\left(f \uparrow_{\hat{\mathbb{R}}}: \hat{\mathbb{R}} \rightarrow \hat{\mathbb{R}}\right) \leq h_{\text {top }}(f: \hat{\mathbb{C}} \rightarrow \hat{\mathbb{C}})=\log (d) .
$$

It would be also useful to know the dimension of the symmetry locus; this is the content of the next proposition. See [MSW17] for a more refined version that determines the dimension of the locus in $\mathcal{M}_{d}(\mathbb{C})$ of rational maps with a prescribed automorphism group.

Proposition 2.4. For all $d \geq 2$ the dimension of the symmetry locus (2.3) is $d-1$ and, as a subvariety of $\mathcal{M}_{d}$ (which is a variety over $\mathbb{Q}$ ), it has a model over $\mathbb{Q}$ (so makes sense to talk of its $\mathbb{R}$-points). Moreover, $\operatorname{dim}_{\mathbb{R}} \mathcal{S}^{\prime}=\operatorname{dim}_{\mathbb{R}} \mathcal{S}(\mathbb{R})=d-1$.

Proof. Let $f \in \mathbb{C}(z)$ be a rational map admitting a non-trivial automorphism $\alpha \in$ $\mathrm{PGL}_{2}(\mathbb{C})$. Since Aut $(f)$ is finite ( Sil07, Proposition 4.65]), after conjugation with an appropriate Möbius map one may assume that the cyclic subgroup generated by $\alpha$ has a generator of the form $\omega_{n}:=z \mapsto \mathrm{e}^{\frac{2 \pi \mathrm{i}}{n}} z$ where $n \geq 2$. The elements of $\operatorname{Rat}_{d}(\mathbb{C})$ commuting with $\omega_{n}$ form the following union:

$$
\bigcup_{0 \leq r \leq n}\left\{\frac{\sum_{0 \leq i \leq d, n \mid i-r-1} a_{i} z^{i}}{\sum_{0 \leq i \leq d, n \mid i-r} b_{i} z^{i}} \in \operatorname{Rat}_{d}(\mathbb{C})\right\} .
$$

Observe that $n$ cannot exceed $d+1$ because otherwise, the set above will be vacuous: there must be $i, j \in\{0, \ldots, d\}$ with both $i-r-1$ and $j-r$ divisible by $n$ and these two numbers differ by at most $d+1$. The dimension of each of the sets in this union is at most $d$ : the number of coefficients $a_{i}, b_{i}$ appearing is precisely $d+1$ for $n=2$ and at most $2\left[\frac{d+1}{3}\right] \leq d+1$ for $n \geq 3$. Thus the projection from the affine space of coefficients into the quasi-projective variety $\operatorname{Rat}_{d}(\mathbb{C})$ yields a $d$-dimensional subset of $\operatorname{Rat}_{d}(\mathbb{C})$. There is one degree of freedom due to conjugation with scaling maps which preserves the forms appeared above. Hence the symmetry locus $\mathcal{S}(\mathbb{C})$ is of dimension at most $d-1$. Indeed, the equality is achieved; otherwise the generic fiber of the projection map from the aforementioned $d$-dimensional subspace of $\operatorname{Rat}_{d}(\mathbb{C})$ into $\mathcal{M}_{d}(\mathbb{C})$ is of dimension at least two. This means that there is a rational map $f \in \mathbb{C}(z)$ commuting with an $\omega_{n}$ such that for any $\alpha$ from a two-dimensional subset $Z$ of $\mathrm{PGL}_{2}(\mathbb{C}), \alpha \circ f \circ \alpha^{-1}$ commute with $\omega_{n}$ as well. In particular, there is a morphism

$$
\left\{\begin{array}{l}
Z \rightarrow \operatorname{Aut}(f) \\
\alpha \mapsto \alpha^{-1} \circ \omega_{n} \circ \alpha
\end{array}\right.
$$

from the two-dimensional variety $Z$ into the finite set Aut $(f)$. So the morphism has to be constant over a two-dimensional subvariety which cannot be the case as 
the centralizer of $\omega_{n}$ in $\mathrm{PGL}_{2}(\mathbb{C})$ is the subgroup of scaling maps $z \mapsto k z$ which is one-dimensional.

For the last part, notice that according to the above union, $\mathcal{S}(\mathbb{C})$ is the image of the algebraic set

$$
\begin{aligned}
\bigcup_{2 \leq n \leq d+1} \bigcup_{0 \leq r \leq n}\left\{\left[a_{0}: \cdots: a_{d}: b_{0}: \cdots: b_{d}\right]\right. \\
\left.\quad \in \operatorname{Rat}_{d}(\mathbb{C}) \subset \mathbb{P}^{2 d+1}(\mathbb{C}) \mid a_{i}=0 \text { if } n \nmid i-r-1 ; b_{i}=0 \text { if } n \nmid i-r\right\}
\end{aligned}
$$

defined over the rationals.

Finally, notice that the dimension count for $\mathcal{S}(\mathbb{C})$ implies upper bounds for dimensions of real subvarieties $\mathcal{S}^{\prime} \subseteq \mathcal{S}(\mathbb{R})$ :

$$
\operatorname{dim}_{\mathbb{R}} \mathcal{S}^{\prime} \leq \operatorname{dim}_{\mathbb{R}} \mathcal{S}(\mathbb{R}) \leq \operatorname{dim}_{\mathbb{C}} \mathcal{S}(\mathbb{C})=d-1 .
$$

It is not hard to show that the equality is achieved here: The complex dimension of $\mathcal{S}(\mathbb{C})$ coincides with the real dimension of its real locus $\mathcal{S}(\mathbb{R})$ provided that the complex variety $\mathcal{S}(\mathbb{C})$ has a smooth $\mathbb{R}$-point in a highest dimensional irreducible component. For a generic choice of complex or real numbers $a_{2 k+1}$ and $b_{2 k}$, the automorphism group of the rational map

$$
\frac{\sum_{0<2 k+1 \leq d} a_{2 k+1} z^{2 k+1}}{\sum_{0 \leq 2 k \leq d} b_{2 k} z^{2 k}}
$$

is generated by $z \mapsto-z$ that on coefficients acts via $a_{2 k+1} \mapsto-a_{2 k+1}$ and $b_{2 k} \mapsto$ $b_{2 k}$. Hence, assuming that these numbers are furthermore positive, we get a $d-$ dimensional submanifold of $\operatorname{Rat}_{d}(\mathbb{R})$ that bijects onto a $(d-1)$-dimensional real submanifold of the set of smooth points of $\mathcal{S}(\mathbb{C})$ which is a subset of $\mathcal{S}^{\prime}$ because every point of it represents the conjugacy class of a real map. Therefore, $\operatorname{dim}_{\mathbb{R}} \mathcal{S}(\mathbb{R}) \geq$ $\operatorname{dim}_{\mathbb{R}} \mathcal{S}^{\prime} \geq d-1$.

2.2. Blaschke products. The rational maps we are interested in are those with real coefficients; or equivalently, those that preserve the real circle $\hat{\mathbb{R}}$. But under a suitable Möbius change of coordinates (e.g. the Cayley transform) $\hat{\mathbb{R}}$ can be identified with the unit circle; and in studying holomorphic maps preserving the unit circle Blaschke products come up naturally. In the next proposition, we use Blaschke products to investigate the domain of the function $h_{\mathbb{R}}$ from Definition 2.3. compare with Bro76].

Proposition 2.5. The domain $\mathcal{M}_{d}^{\prime}-\mathcal{S}^{\prime} \subset \mathcal{M}_{d}(\mathbb{C})$ of the real entropy function (2.4) is an irreducible real variety of dimension $2 d-2$. Nevertheless, in its analytic topology, it decomposes to $d+1$ connected components of the same dimension corresponding to topological degrees of self-maps of the circle $\hat{\mathbb{R}}=\mathbb{R} \cup\{\infty\}$ that elements of $\operatorname{Rat}_{d}(\mathbb{R})$ induce:

$$
\mathcal{M}_{d}^{\prime}-\mathcal{S}^{\prime}=\bigsqcup_{-d \leq s \leq d, 2 \mid d-s}\left(\mathcal{M}_{d, s}^{\prime}-\mathcal{S}^{\prime}\right)
$$

Here, $\mathcal{M}_{d, s}^{\prime}$ is the subspace of complex conjugacy classes of degree d real rational maps $f$ for which the topological degree of the restriction $f \uparrow_{\hat{\mathbb{R}}}: \hat{\mathbb{R}} \rightarrow \hat{\mathbb{R}}$ is s.

Proof. We have

$$
\operatorname{dim}_{\mathbb{R}} \mathcal{M}_{d}^{\prime} \leq \operatorname{dim}_{\mathbb{R}} \mathcal{M}_{d}(\mathbb{R}) \leq \operatorname{dim}_{\mathbb{C}} \mathcal{M}_{d}(\mathbb{C})=2 d-2 .
$$


The surjective morphism $\operatorname{Rat}_{d}(\mathbb{R}) / \mathrm{PGL}_{2}(\mathbb{R}) \rightarrow \mathcal{M}_{d}^{\prime}$ is injective on the Zariski open subset of classes without twists (e.g. away from the preimage of the closed subvariety $\mathcal{S}^{\prime}$ ). The projection also indicates that $\mathcal{M}_{d}^{\prime}$ is irreducible, being a surjective image of $\operatorname{Rat}_{d}(\mathbb{R})$ which is irreducible itself as it is the complement of the resultant hypersurface in $\mathbb{P}^{2 d+1}(\mathbb{R})$. Therefore, $\mathcal{M}_{d}^{\prime}$ is irreducible in the Zariski topology; and is of real dimension $2 d-2$. The same statements hold for its Zariski open subset $\mathcal{M}_{d}^{\prime}-\mathcal{S}^{\prime}$

Since the field $\mathbb{R}$ is not algebraically closed, the irreducibility of $\operatorname{Rat}_{d}(\mathbb{R})$ or $\mathcal{M}_{d}^{\prime}-\mathcal{S}^{\prime}$ does not guarantee their connectedness in the analytic topology. As a matter of fact, maps in $\operatorname{Rat}_{d}(\mathbb{R})$ whose restrictions to the real circle have different topological degrees cannot lie in the same connected component. To see this, notice that the integer-valued function

$$
\operatorname{deg}_{\text {top }}: \mathcal{M}_{d}^{\prime}-\mathcal{S}^{\prime} \rightarrow\{-d, \ldots, 0, \ldots, d\}
$$

is continuous because the topological degree can be expressed as an integral over the circle $\hat{\mathbb{R}}=\mathbb{R} \cup\{\infty\}$. Hence the level sets of $\operatorname{deg}_{\text {top }}$ are disjoint unions of connected components of $\operatorname{Rat}_{d}(\mathbb{R})$. The topological degree of a circle map is preserved under conjugation by a diffeomorphism of the circle and Proposition 2.2 implies that if real maps $f, g$ give rise to the same class in $\mathcal{M}_{d}(\mathbb{C})$ away from $\mathcal{S}^{\prime}$, then they are conjugate by a Möbius transformation that preserves $\hat{\mathbb{R}}$. Therefore, the topological degree descends to a continuous function

$$
\operatorname{deg}_{\text {top }}: \mathcal{M}_{d}^{\prime}-\mathcal{S}^{\prime} \rightarrow\{-d, \ldots, 0, \ldots, d\}
$$

any level set $\mathcal{M}_{d, s}^{\prime}-\mathcal{S}^{\prime}$ of which is a union of connected components of $\mathcal{M}_{d}^{\prime}-\mathcal{S}^{\prime}$. Here

$$
\mathcal{M}_{d, s}^{\prime}:=\left\{\langle f\rangle \in \mathcal{M}_{d}(\mathbb{C}) \mid f \in \operatorname{Rat}_{d}(\mathbb{R}), \operatorname{deg}_{\text {top }}\left(f \uparrow_{\hat{\mathbb{R}}}: \hat{\mathbb{R}} \rightarrow \hat{\mathbb{R}}\right)=s\right\}
$$

as defined in the statement of the proposition.

We next show that $\mathcal{M}_{d, s}^{\prime}$ is non-vacuous if and only if $d$ and $s \in\{-d, \ldots, 0, \ldots, d\}$ have the same parity and for any such $s$ the subspace $\mathcal{M}_{d}^{\prime}-\mathcal{S}^{\prime}$ is connected. This will establish the partition (2.5) of $\mathcal{M}_{d}^{\prime}-\mathcal{S}^{\prime}$ to its connected components and will conclude the proof.

Let us proceed with a parametrization of degree $d$ real rational maps with $\operatorname{deg}_{\text {top }}=s$. It is more convenient to exchange $\hat{\mathbb{R}}$ with $\{z \in \mathbb{C}|| z \mid=1\}$ via the Cayley transform

$$
\left\{\begin{array}{l}
\text { the unit circle } \rightarrow \text { the real circle } \\
z \mapsto x=\mathrm{i} \frac{1+z}{1-z}
\end{array}\right.
$$

and concentrate on degree $d$ rational maps $f \in \mathbb{C}(z)$ that keep the unit circle $\{z \in \mathbb{C}|| z \mid=1\}$ invariant instead. The reflection $z \mapsto \frac{1}{\bar{z}}$ with respect to the unit circle commutes with such an $f$, so the roots and the poles of $f$ on the Riemann sphere occur in (root, pole) pairs like $\left(q, \frac{1}{\bar{q}}\right)$ where $q$ is away from the unit circle. Therefore, assuming that $f$ has $k$ roots $a_{1}, \ldots, a_{k}$ inside the unit circle and $d-$ $k$ roots $b_{1}, \ldots, b_{d-k}$ outside of it (both written by multiplicity), its poles would be reciprocals of $\overline{a_{1}}, \ldots, \overline{a_{k}}$ outside the unit circle and reciprocals of $\overline{b_{1}}, \ldots, \overline{b_{d-k}}$ 
inside it. The rational maps $f(z)$ is thus a scalar multiple of the Blaschke product

$$
\prod_{i=1}^{k}\left(\frac{z-a_{i}}{1-\bar{a}_{i} z}\right) \prod_{j=1}^{d-k}\left(\frac{z-b_{j}}{1-\bar{b}_{j} z}\right)
$$

that preserves the unit circle as well. We conclude that $f(z)$ has a unique description as

$$
\begin{array}{r}
f(z)=\mathrm{e}^{2 \pi \mathrm{i} c} \prod_{i=1}^{k}\left(\frac{z-a_{i}}{1-\bar{a}_{i} z}\right) \prod_{j=1}^{d-k}\left(\frac{z-b_{j}}{1-\bar{b}_{j} z}\right) \quad\left(\left|a_{1}\right|, \ldots,\left|a_{k}\right|<1 ;\left|b_{1}\right|, \ldots,\left|b_{d-k}\right|\right. \\
>1 ; c \in \mathbb{R} / \mathbb{Z}) .
\end{array}
$$

The topological degree $s$ of the restriction $\left.f\right|_{\{|z|=1\}}:\{|z|=1\} \rightarrow\{|z|=1\}$ of the map in (2.8) is $k-(d-k)=2 k-d$ by an easy application of the argument principle. We observe that $k=\frac{d+s}{2}$ and an integer $s$ from $\{-d, \ldots, 0, \ldots, d\}$ can be realized as the topological degree of the restriction precisely when its parity is the same as that of $d$. Now it is easy to verify that there are $d+1$ choices for $s$.

Fixing such an $s$, putting $k$ to be $\frac{d+s}{2}$ in (2.8) and then varying $a_{i}$ 's, $b_{j}$ 's and $c$ respectively inside, outside and on the unit circle, and finally conjugating with the Cayley transform parametrizes a submanifold of $\operatorname{Rat}_{d}(\mathbb{R})$ diffeomorphic to $\mathbb{R}^{2 d} \times S^{1}$ which is the subspace of real rational maps of degree $d$ with $\operatorname{deg}_{\text {top }}=s$. This full-dimensional submanifold of $\operatorname{Rat}_{d}(\mathbb{R})$ surjects onto the subspace $\mathcal{M}_{d, s}^{\prime}$ of $\mathcal{M}_{d}^{\prime}$ appeared in (2.6); a subspace that is therefore connected and of codimension zero. Finally, we argue that removing $\mathcal{S}^{\prime}$ in (2.5) cannot affect the connectedness of $\mathcal{M}_{d, s}^{\prime}$ : If $d \geq 3$, invoking Proposition 2.4, the codimension of $\mathcal{M}_{d, s}^{\prime} \cap \mathcal{S}^{\prime}$ in $\mathcal{M}_{d, s}^{\prime}$ is

$$
(2 d-2)-(d-1) \geq 2 .
$$

Taking preimages under $\operatorname{Rat}_{d}(\mathbb{R}) \rightarrow \mathcal{M}_{d}^{\prime}$, we deduce that in the $\operatorname{deg}_{\text {top }}=s$ component of Rat $_{d}$ - which is a manifold - the closed subset of maps with non-trivial automorphisms is of codimension at least two, and hence the complement - which surjects onto $\mathcal{M}_{d, s}^{\prime}-\mathcal{S}^{\prime}-$ is connected. For $d=2$, we rely on the results of [Mil93]: $\mathcal{S}^{\prime}=\mathcal{S}(\mathbb{R})$ is precisely the curve in $\mathcal{M}_{2}^{\prime}=\mathcal{M}_{2}(\mathbb{R})=\mathbb{R}^{2}$ that separates $\mathcal{M}_{2,+2}^{\prime}$, $\mathcal{M}_{2,-2}^{\prime}$ and $\mathcal{M}_{2,0}^{\prime}$; see [Mil93, fig. 15].

Example 2.6. For a given $0 \neq s \in\{ \pm 1, \ldots, \pm d\}$ with $s \equiv d(\bmod 2)$, the Blaschke product

$$
\begin{array}{r}
f(z)=\mathrm{e}^{2 \pi \mathrm{i} c} \prod_{i=1}^{\frac{d+s}{2}}\left(\frac{z-a_{i}}{1-\bar{a}_{i} z}\right) \prod_{j=1}^{\frac{d-s}{2}}\left(\frac{z-b_{j}}{1-\bar{b}_{j} z}\right)\left(\left|a_{1}\right|, \ldots,\left|a_{\frac{d+s}{2}}\right|<1 ;\left|b_{1}\right|, \ldots,\left|b_{\frac{d-s}{2}}\right|\right. \\
>1 ; c \in \mathbb{R} / \mathbb{Z})
\end{array}
$$

appeared in the proof of Proposition 2.5 induces a (unramified) degree $s$ covering of $|z|=1$ provided that $a_{i}$ 's and $b_{j}$ 's are sufficiently close to 0 and $\infty$ respectively. Conjugation with the Cayley transform (2.7) then yields a degree $d$ rational map that restricts to a degree $s$ cover $\hat{\mathbb{R}} \rightarrow \hat{\mathbb{R}}$; thus a degree $d$ real map of real entropy $\log (|s|)$. For generic choices of $a_{i}$ 's and $b_{j}$ 's, the corresponding map $f$ will be away 
from the symmetry locus. We conclude that $h_{\mathbb{R}}$ is $\log (|s|)$ over some non-empty analytic open subset of the connected component $\mathcal{M}_{d, s}^{\prime}$.

The dynamics of (2.9) on the unit circle is well understood once the map is expanding (e.g. as $a_{i} \rightarrow 0$ and $b_{j} \rightarrow \infty$ ) since then, according to [Shu69], it should be conjugate to $z \mapsto z^{s}$. Such expanding maps do occur once $|s| \geq 2$. Notice that for a Blaschke product $f$ from (2.9) which is expanding on the unit circle, the Julia set indeed contains the unit circle. Finally, we point out that although when $|s|<d$ the unit circle does not capture all of the entropy of the ambient endomorphism of the Riemann sphere, the dynamics on the circle demonstrates certain "rigidity" here in the sense that once two expanding circle maps of the form (2.9) are absolutely continuously conjugate, the ambient rational maps must be Möbius conjugate [SS85, Theorem 4].

For $s=1$ and $d$ odd, this type of Blaschke product is used in a construction of rational maps with Herman rings; see [Mil06a, §15]. In such a situation,

$$
f \nmid\{|z|=1\}:\{|z|=1\} \rightarrow\{|z|=1\}
$$

would be an orientation-preserving analytic diffeomorphism whose rotation number $\rho$ can be any desired element in $\mathbb{R} / \mathbb{Z}$ after a suitable adjustment of $c$ in (2.9) Mil06a, §15, Lemma 15.3]. The entropy of this subsystem is then zero. If $\rho$ is rational, then every orbit on the circle converges to a periodic orbit; and the only possible Julia points on the invariant circle are parabolic points.

For irrational $\rho$, by Denjoy's theorem there is a topological conjugacy between $f \nmid\{|z|=1\}:\{|z|=1\} \rightarrow\{|z|=1\}$ and the irrational rotation $t \in \mathbb{R} / \mathbb{Z} \mapsto t+\rho \in \mathbb{R} / \mathbb{Z}$; in particular, every single orbit of this subsystem is dense. So the unit circle is entirely included in either the Julia set or the Fatou set. The latter situation occurs precisely when the diffeomorphism $f\lceil\{|z|=1\}:\{|z|=1\} \rightarrow\{|z|=1\}$ is real analytically linearizable in which case the circle is in a Herman ring. There is a complete classification based on Diophantine properties due to Yoccoz of rotation numbers for which the existence of a real analytic linearization is guaranteed [Yoc02]. For irrational rotation numbers $\rho$ that are "too well approximated" by rational numbers, there are real analytic diffeomorphisms of the unit circle of rotation number $\rho$ that do not admit even $C^{\infty}$ linearizations. Hence if in (2.9) we fix $a_{1}, \ldots, a_{\frac{d+1}{2}}$ near 0 and $b_{1}, \ldots, b_{\frac{d-1}{2}}$ near $\infty$ and then adjust $c$ so that we get such a rotation number for the induced self-diffeomorphism of $|z|=1$, then all points on the unit circle would be Julia despite the fact that the real entropy vanishes.

2.3. Properties of $h_{\mathbb{R}}: \mathcal{M}_{d}^{\prime}-\mathcal{S}^{\prime} \rightarrow[0, \log (d)]$. We now prove the first part of Theorem 1.1

Proposition 2.7. For any $d \geq 2$, the function $h_{\mathbb{R}}: \mathcal{M}_{d}^{\prime}-\mathcal{S}^{\prime} \rightarrow[0, \log (d)]$ is surjective and continuous (in the analytic topology).

Proof. Fix an $s \in\{-d, \ldots, 0 \ldots, d\}$ with $s \equiv d(\bmod 2)$. As observed in the proof of Proposition 2.5 and also in Example 2.6, the component $\operatorname{deg}_{\text {top }}=s$ of $\operatorname{Rat}_{d}(\mathbb{R})$ can be identified with the space of Blaschke products

$$
\begin{array}{r}
\mathrm{e}^{2 \pi \mathrm{i} c} \prod_{i=1}^{\frac{d+s}{2}}\left(\frac{z-a_{i}}{1-\overline{a_{i} z}}\right) \prod_{j=1}^{\frac{d-s}{2}}\left(\frac{z-b_{j}}{1-\overline{b_{j} z}}\right)\left(\left|a_{1}\right|, \ldots,\left|a_{\frac{d+s}{2}}\right|<1 ;\left|b_{1}\right|, \ldots,\left|b_{\frac{d-s}{2}}\right|\right. \\
>1 ; c \in \mathbb{R} / \mathbb{Z})
\end{array}
$$


appeared in (2.9). A convergence of the parameters of the above product inside

$$
\{|z|<1\}^{\frac{d+s}{2}} \times\{|z|>1\}^{\frac{d-s}{2}} \times \mathbb{R} / \mathbb{Z}
$$

results in the uniform convergence of the corresponding holomorphic functions over some thin enough annulus around the unit circle $|z|=1$ and therefore, the convergence of the induced maps of the unit circle in the $C^{\infty}$ topology. The continuity claim follows immediately from [Mis95]: in the $C^{1}$ topology, the topological entropy is continuous for circle or interval maps of bounded modality. Consequently, the real entropy is a continuous function on the space $\operatorname{Rat}_{d}(\mathbb{R})$ of real rational maps of degree $d$. This function, after being factored through the local homeomorphism from the open subset of maps without Möbius symmetries in $\operatorname{Rat}_{d}(\mathbb{R})$ onto $\mathcal{M}_{d}^{\prime}-\mathcal{S}^{\prime}$, descends to the function $h_{\mathbb{R}}: \mathcal{M}_{d}^{\prime}-\mathcal{S}^{\prime} \rightarrow[0, \log (d)]$ which is thus continuous as well.

Because of the continuity, to obtain the surjectivity of $h_{\mathbb{R}}$ it suffices to construct a family of real rational maps of degree $d$ parametrized over a connected space for which the real entropy gets arbitrarily close to both extremes 0 and $\log (d)$. We invoke the result [MT00, Theorem 3.2] that allows us to parametrize the class of boundary-anchored polynomial interval maps of full modality via their critical values:

Given numbers $v_{1}, \ldots, v_{d-1} \in[-1,1]$ with $(-1)^{i}\left(v_{i}-v_{i-1}\right)>0$ for every $0<i \leq d$ where $v_{0}:=1$ and $v_{d}:=(-1)^{d}$, there is a unique boundaryanchored polynomial map $f:[-1,1] \rightarrow[-1,1]$ of degree $d$ that has distinct critical points

$$
-1<c_{1}<\cdots<c_{d-1}<1
$$

such that $f\left(c_{i}\right)=v_{i}$ for all $0<i<d$ and $f(-1)=v_{0}, f(1)=v_{d}$ on the boundary.

The space of these tuples $\left(v_{1}, \ldots, v_{d-1}\right) \in[-1,1]^{d-1}$ is obviously connected. As $v_{i} \rightarrow 0^{+}$for $0<i<d$ even and $v_{i} \rightarrow 0^{-}$for $0<i<d$ odd, the corresponding maps tend to $x \mapsto(-x)^{d}$ whose real entropy is zero, whereas when $v_{i}=(-1)^{i}$ for each $0<i<d$, the corresponding degree $d$ polynomial map $f:[-1,1] \rightarrow[-1,1]$ would have $d$ surjective monotonic pieces; so the iterate $f^{\circ n}$ needs to have $d^{n}$ laps and therefore the exponential growth rate of modality of iterates is $\log (d)$.

Remark 2.8. Notice that for the family exhibited at the end of the preceding proof the topological degree $s$ is zero for $d$ even and is -1 for $d$ odd. Indeed, although surjective on $\mathcal{M}_{d}^{\prime}-\mathcal{S}^{\prime}, h_{\mathbb{R}}$ is not surjective on all constituent parts of $\mathcal{M}_{d}^{\prime}-\mathcal{S}^{\prime}$ that appeared in (2.5); a well known result from [MP77] asserts that for a $C^{1}$ self-map of a compact connected differentiable oriented manifold of degree $s$ the topological entropy is at least $\log (\max (|s|, 1))$.

2.4. Comparing $\mathcal{M}_{d}^{\prime}$ with the real locus $\mathcal{M}_{d}(\mathbb{R})$. Next, we elaborate more on the domain $\mathcal{M}_{d}^{\prime}-\mathcal{S}^{\prime}=\mathcal{M}_{d}^{\prime}-\mathcal{S}(\mathbb{R})$ of $h_{\mathbb{R}}$ in (2.4); we will establish that it differs from $\mathcal{M}_{d}(\mathbb{R})-\mathcal{S}(\mathbb{R})$ by an irreducible component which is relevant only in odd degrees. The subspace $\mathcal{M}_{d}^{\prime}$ defined in (2.1) is formed by the conjugacy classes of elements of $\operatorname{Rat}_{d}(\mathbb{C})$ for which $\mathbb{R}$ is a field of definition. On the other hand, $\mathbb{R}$ points of the moduli space $\mathcal{M}_{d}$ are Möbius conjugacy classes of elements of $\operatorname{Rat}_{d}(\mathbb{C})$ whose field of moduli is included in $\mathbb{R}$; see [Sil07, $\S \S 4.4,4.10]$ for the background material. So $\mathcal{M}_{d}^{\prime} \subseteq \mathcal{M}_{d}(\mathbb{R})$ and in particular $\mathcal{M}_{d}^{\prime}-\mathcal{S}^{\prime} \subseteq \mathcal{M}_{d}(\mathbb{R})-\mathcal{S}(\mathbb{R})$. The latter containment (and consequently the former) can indeed be strict: for $d=2 k+1$ the 
automorphism group of the rational map $\phi(z):=\mathrm{i}\left(\frac{z-1}{z+1}\right)^{2 k+1}$ is trivial and its field of moduli is $\mathbb{Q}$ while it cannot be defined over the reals; see Sil07, Exercise 4.39, Example 4.85]. It is worth noting that this question of "FoM vs. FoD" is relevant only when $d$ is odd [Sil07, Theorem 4.92].

Let us try to see how the real variety $\mathcal{M}_{d}(\mathbb{R})-\mathcal{S}(\mathbb{R})$ is related to its (possibly proper) Zariski closed subset $\mathcal{M}_{d}^{\prime}-\mathcal{S}(\mathbb{R})=\mathcal{M}_{d}^{\prime}-\mathcal{S}^{\prime}$. We claim that classes in the complement $\mathcal{M}_{d}(\mathbb{R})-\left(\mathcal{S}(\mathbb{R}) \cup \mathcal{M}_{d}^{\prime}\right)=\mathcal{M}_{d}(\mathbb{R})-\left(\mathcal{S}(\mathbb{C}) \cup \mathcal{M}_{d}^{\prime}\right)$ can be represented by antipodal maps; i.e. maps which commute with the anti-holomorphic involution $\gamma(z):=-\frac{1}{\bar{z}}$. Notice that the example $\phi(z)=\mathrm{i}\left(\frac{z-1}{z+1}\right)^{d}$ for $d$ odd that appeared before is indeed antipodal. For more on the dynamics of antipodal-preserving maps see [BBM15]. They are not relevant to our treatment of entropy as a generic map of this class does not preserve any circle.

Proposition 2.9. The real subvariety $\mathcal{M}_{d}^{\prime}$ of $\mathcal{M}_{d}(\mathbb{C})$ coincides with the real locus $\mathcal{M}_{d}(\mathbb{R})$ of the moduli space for $d$ even while for $d$ odd, the latter is reducible and has $\mathcal{M}_{d}^{\prime}$ and the $(2 d-2)$-dimensional real subvariety of conjugacy classes of antipodal-preserving maps as irreducible components. Any other irreducible component of $\mathcal{M}_{d}(\mathbb{R})$ has to be contained in the symmetry locus $\mathcal{S}(\mathbb{R})$ and hence is of dimension at most $d-1$.

Proof. Invoking [Sil07, Proposition 4.86], the obstruction to the field of moduli $\mathbb{R}$ being a field of definition for a map $f \in \operatorname{Rat}_{d}(\mathbb{C})$ with $\operatorname{Aut}(f)=\{\mathbf{1}\}$ is encoded by the Galois cohomology class determined by the cocycle Gal $(\mathbb{C} / \mathbb{R}) \rightarrow \mathrm{PGL}_{2}(\mathbb{C})$ defined by $\sigma \mapsto \alpha$ with $\sigma$ being the complex conjugation and $\alpha$ a Möbius transformation with $\alpha \circ f \circ \alpha^{-1}=\bar{f}$ that therefore satisfies the cocycle condition $\alpha \circ \bar{\alpha}=1$. These transformations have to be considered modulo modification via a 1-coboundary, i.e. replacing $\alpha$ with $u \circ \alpha \circ \bar{u}^{-1}$ that amounts to replacing $f$ with $\bar{u} \circ f \circ \bar{u}^{-1}$ from the same conjugacy class. But the group 1

$$
H^{1}\left(\mathrm{Gal}(\mathbb{C} / \mathbb{R}), \mathrm{PGL}_{2}(\mathbb{C})\right) \cong H^{2}\left(\operatorname{Gal}(\mathbb{C} / \mathbb{R}), \mathbb{C}^{\times}\right) \cong \operatorname{Br}(\mathbb{R})
$$

is of order two and is thus generated by any 1-cocycle non-cohomologous to a coboundary. An example of such is the 1-cocycle associated to the rational map $\phi(z)=\mathrm{i}\left(\frac{z-1}{z+1}\right)^{2 k+1}$ appeared before:

$$
-\frac{1}{\phi\left(\frac{-1}{z}\right)}=-\frac{1}{\mathrm{i}\left(\frac{-\frac{1}{z}-1}{-\frac{1}{z}+1}\right)^{2 k+1}}=-\mathrm{i}\left(\frac{z-1}{z+1}\right)^{2 k+1}=\bar{\phi}(z) ;
$$

that furnishes us with the 1-cocycle determined by $\alpha(z)=-\frac{1}{z}$. It is easy to verify that this is not 1-coboundary; that is, not in the form of $u \circ \bar{u}^{-1}$ for another Möbius transformation; see [Sil07, p. 209] for details. Hence $\mathcal{M}_{d}(\mathbb{R})-\left(\mathcal{S}(\mathbb{R}) \cup \mathcal{M}_{d}^{\prime}\right)$ is precisely the subset of classes in $\mathcal{M}_{d}(\mathbb{R})-\mathcal{S}(\mathbb{R})$ which admit a representative $f \in \mathbb{C}(z)$ with $\bar{f}\left(-\frac{1}{z}\right)=-\frac{1}{f(z)}$. Applying the complex conjugation map to both sides, this condition means that $f$ commutes with the anti-holomorphic involution $\gamma(z)=-\frac{1}{\bar{z}}$, a Zariski closed condition over the reals cutting out the antipodalpreserving locus of the moduli space. Notice that the constraint automatically guarantees that the field of moduli is inside $\mathbb{R}$ as the complex conjugate map $\bar{f}$ is Möbius conjugate to $f$ via $z \mapsto-\frac{1}{z}$. But there might be such maps which cannot be

\footnotetext{
${ }^{1}$ Here $\operatorname{Br}(\mathbb{R})$ denotes the Brauer group of the field of real numbers.
} 
defined over the reals; e.g., the example $\phi(z)=\mathrm{i}\left(\frac{z-1}{z+1}\right)^{d}$ for $d$ odd. Consequently, the real variety $\mathcal{M}_{d}(\mathbb{R})-\mathcal{S}(\mathbb{R})$ is the union of $\mathcal{M}_{d}^{\prime}-\mathcal{S}(\mathbb{R})$ - that as discussed before in $\$ 2.2$ is irreducible and of dimension $2 d-2$ - and the antipodal locus.

Here is a simple dimension count for the antipodal-preserving locus in $\mathcal{M}_{d}(\mathbb{C})$. Picking a degree $d$ map $f$ which commutes with $\gamma: z \mapsto-\frac{1}{\bar{z}}$, after a Möbius conjugation, without any loss of generality we may assume that the roots of $f$ lie in the finite plane. If $q$ is a root of $f,-\frac{1}{\bar{q}}$ has to be a pole. Thus roots and poles can be coupled in pairs such as $\left(q_{i},-\frac{1}{\bar{q}_{i}}\right)$. We conclude that $f(z)$ is a scalar multiple of a function in the form of $\prod_{i=1}^{d} \frac{z-q_{i}}{1+\bar{q}_{i} z}$. Now $f \circ \gamma=\gamma \circ f$ is satisfied for a multiple of such a product if and only if $d$ is odd and the scalar factor is of norm one. This argument indicates that any antipodal-preserving map of odd degree $d$, after a suitable conjugation, can be uniquely written as

$$
u \prod_{i=1}^{d} \frac{z-q_{i}}{1+\bar{q}_{i} z}
$$

where $|u|=1$ and $q_{i}$ 's are complex numbers with $q_{i} \overline{q_{j}} \neq-1$. This parametrizes a connected subspace of real dimension $2 d+1$ of $\operatorname{Rat}_{d}(\mathbb{C})$ that projects onto the antipodal-preserving locus in $\mathcal{M}_{d}(\mathbb{C})$. Consequently, like $\mathcal{M}_{d}^{\prime}$, the antipodal locus is irreducible as well, being a surjective image of the irreducible real algebraic subset $S^{1} \times\left(\mathbb{C}^{\times}\right)^{d}$ of $\mathbb{A}^{2 d+1}(\mathbb{R})$. Let us find the dimension of the intersection of the whole conjugacy class of a generic map of this kind with the antipodal-preserving locus in $\operatorname{Rat}_{d}(\mathbb{C})$. Given an antipodal map $f$ with $\operatorname{Aut}(f)=\{\mathbf{1}\}$, suppose for an $\alpha \in \mathrm{PGL}_{2}(\mathbb{C})$ the map $\alpha \circ f \circ \alpha^{-1}$ is antipodal too. Then one can write:

$$
\gamma \circ\left(\alpha \circ f \circ \alpha^{-1}\right) \circ \gamma^{-1}=\alpha \circ f \circ \alpha^{-1}=\alpha \circ\left(\gamma \circ f \circ \gamma^{-1}\right) \alpha^{-1},
$$

which indicates that the Möbius transformation $(\alpha \circ \gamma)^{-1} \circ(\gamma \circ \alpha)$ is an automorphism of $f$ and thus $\alpha$ commutes with $\gamma$. It is not hard to verify that any Möbius transformation commuting with $z \mapsto-\frac{1}{\bar{z}}$ can be uniquely written either as $z \mapsto \frac{z+a \mathrm{e}^{2 \pi \mathrm{i} r}}{a \mathrm{e}^{2 \pi \mathrm{i} s} z-\mathrm{e}^{2 \pi \mathrm{i}(r+s)}}$ with $r, s \in \mathbb{R} / \mathbb{Z}, a>0$ or in one of forms $z \mapsto v z, z \mapsto \frac{v}{z}$ where $v$ lies on the unit circle. We conclude that the space of Möbius transformation commuting with the antipodal involution is of real dimension three and thus the antipodal locus in $\mathcal{M}_{d}(\mathbb{C})$ is of dimension $(2 d+1)-3=2 d-2$.

For more on $\mathcal{M}_{d}(\mathbb{R})$, see $\mathrm{HQ} 15$.

\section{Rigidity OF REAL ENTROPY}

Equipped with the definition of the function $h_{\mathbb{R}}: \mathcal{M}_{d}^{\prime}-\mathcal{S}^{\prime} \rightarrow[0, \log (d)]$ from \$2 we prove Theorem 1.1 in this section. Away from the antipodal and symmetry loci, $\mathcal{M}_{d}^{\prime}$ can be thought of as the set of fixed points of the involution $\langle f\rangle \mapsto\langle\bar{f}\rangle$ induced by conjugating coefficients in $\operatorname{Rat}_{d}(\mathbb{C})$. For future references, we record this involution as acting not only on rational maps but on functions defined on the Riemann sphere:

Definition 3.1. For any function $h: \hat{\mathbb{C}} \rightarrow \mathbb{C} \cup\{\infty\}$, the function $\tilde{h}: \hat{\mathbb{C}} \rightarrow \mathbb{C} \cup\{\infty\}$ is defined as:

$$
\tilde{h}: z \mapsto \overline{h(\bar{z})}
$$


It is easy to check that $h \mapsto \tilde{h}$ is an involution which respects the ring structure of the set of $\mathbb{C}$-valued functions on the Riemann sphere; takes homeomorphisms to homeomorphisms and finally, it commutes with differential operators $\frac{\partial}{\partial z}$ and $\frac{\partial}{\partial \bar{z}}$ :

$$
\frac{\partial \tilde{h}}{\partial z}=\frac{\widetilde{\partial h}}{\partial z}, \quad \frac{\partial \tilde{h}}{\partial \bar{z}}=\frac{\widetilde{\partial h}}{\partial \bar{z}} .
$$

We now briefly review the theory of quasi-conformal deformations of rational maps developed in MS98. A concise treatment could be found in one of the additional chapters of Ahl06. Let $f$ be an arbitrary real rational function of degree $d$. The quasi-conformal (q.c.) conjugacy class of $f$, denoted by qc $(f)$, consists of those rational maps $g$ which are quasi-conformally conjugate to $f$. The space

$$
\mathrm{M}(f):=\mathrm{qc}(f) / \text { Möbius Equivalence }
$$

of Möbius conjugacy classes of maps in qc $(f)$ is called the moduli space of the rational map $f$ and the natural map $\mathrm{M}(f) \hookrightarrow \mathcal{M}_{d}(\mathbb{C})$ is an injection of complex orbifolds. In analogy with the theory of the moduli spaces of Riemann surfaces, the moduli space $\mathrm{qc}(f)$ is the quotient of a Teichmüller space under a discrete group action:

$\mathrm{T}(f)=\{(g, h) \mid g$ a rational map, $h$ a q.c.-homeomorphism with $h \circ f=g \circ h\} / \sim$ where $\left(g_{1}, h_{1}\right) \sim\left(g_{2}, h_{2}\right)$ if in the conjugacy

$$
g_{2}=\left(h_{2} \circ h_{1}^{-1}\right) \circ g_{1} \circ\left(h_{2} \circ h_{1}^{-1}\right)^{-1}
$$

the quasi-conformal homeomorphism $h_{2} \circ h_{1}^{-1}$ is isotopic to a Möbius transformation through an isotopy which preserves the conjugacy. In particular, $g_{1}, g_{2}$ must be Möbius conjugate. There is an obvious map

$$
\left\{\begin{array}{l}
\mathrm{T}(f) \rightarrow \mathrm{M}(f)=\mathrm{qc}(f) / \text { Möbius Equivalence } \\
{[(g, h)] \mapsto\langle g\rangle}
\end{array}\right.
$$

sending the class $[(g, h)]$ of a pair to the class $\langle g\rangle$. The fiber above the Möbius class of $f$ can be identified with the group of isotopy classes of q.c.-homeomorphisms commuting with $f$ where the isotopy has to remain within this space of q.c.automorphisms as well. The aforementioned group is called the modular group of the rational map $f$ and will be denoted by $\operatorname{Mod}(f)$. Clearly, $\operatorname{Mod}(f)$ acts (from right) on $\mathrm{T}(f)$ by $[(g, h)] \cdot[k]=[(g, h \circ k)]$ and the quotient $\mathrm{T}(f) / \operatorname{Mod}(f)$ can be identified with $\mathrm{M}(f)$ via the projection above. It is known that $\operatorname{Mod}(f)$ is discrete and acts properly discontinuously on $\mathrm{T}(f)$. Furthermore, there is a description of the space $\mathrm{T}(f)$ as a product of ordinary Teichmüller spaces based on the dynamics of $f: \hat{\mathbb{C}} \rightarrow \hat{\mathbb{C}}$; see [MS98, Theorems $2.2 \& 2.3$ ].

There is a naturally defined entropy function $\widetilde{h_{\mathbb{R}}}$ on some appropriate subset of $\mathrm{T}(f)$ sending a class $[(g, h)]$ with $\operatorname{Aut}(g)=\{\mathbf{1}\}$ to $h_{\mathbb{R}}(g)$. This is well defined and fits in the commutative diagram below:

$$
\begin{aligned}
& \{[(g, h)] \in \mathrm{T}(f) \mid g \in \mathbb{R}(z), \operatorname{Aut}(g)=\{\mathbf{1}\}\} \\
& \begin{array}{c}
{[(g, h)] \mapsto\langle g\rangle} \\
\mathcal{M}_{d}^{\prime} \cap \mathrm{M}(f)-\mathcal{S}^{\prime} \longrightarrow \widetilde{h_{\mathbb{R}}} \\
\longrightarrow
\end{array}[0, \log (d)]
\end{aligned}
$$


Suppose $g$ is another real rational map quasi-conformally conjugate to $f$; that is, $(g, h)$ determines a class in $\mathrm{T}(f)$ for a suitable q.c.-homeomorphisms $h$ satisfying $h \circ f=g \circ h$. In order to compare the topological entropies of $f \uparrow_{\hat{\mathbb{R}}}$ and $g \uparrow_{\hat{\mathbb{R}}}$, we need to investigate how the quasi-circle $h(\hat{\mathbb{R}})$ is placed with respect to $\hat{\mathbb{R}}$. Pulling back the complex conjugate map $z \mapsto \bar{z}$ via $h$ yields a reflection $z \mapsto h^{-1}(\overline{h(z)})$ commuting with $f$. This differs from the usual reflection $z \mapsto \bar{z}$ - that also preserves $f \in \mathbb{R}(z)$ - by a q.c. automorphism of $f$ :

$$
u(z):=\overline{h^{-1}(\overline{h(z)})}
$$

a map which satisfies $u(\overline{u(\bar{z})})=z$ or equivalently, using the notation in (3.1)

$$
u^{-1}=\tilde{u}
$$

holds. The homeomorphism $u$ is identity if and only if $\overline{h(\bar{z})}=z$ which in particular indicates that $h$ preserves $\hat{\mathbb{R}}$. Next, we form a continuous map that assigns to a $[(g, h)] \in \mathrm{T}(f)$ (with $g \in \mathbb{R}(z))$ the class in $\operatorname{Mod}(f)$ of the corresponding automorphism $u$ from (3.5). The appropriate domain of definition for such a map turns out to be that of the function $\widetilde{h_{\mathbb{R}}}$ from $(3.4)$ as the following proposition suggests. The proof is straightforward and is left to the reader.

Proposition 3.2. The map

$$
\left\{\begin{array}{l}
\{[(g, h)] \in \mathrm{T}(f) \mid g \in \mathbb{R}(z), \operatorname{Aut}(g)=\{\mathbf{1}\}\} \rightarrow \operatorname{Mod}(f) \\
{[(g, h)] \mapsto\left[u: z \mapsto \overline{h^{-1}(\overline{h(z)})}\right]}
\end{array}\right.
$$

is well defined.

The map (3.7) is obviously continuous, but attains its values in the discrete group $\operatorname{Mod}(f)$, so must be constant on each connected components of the domain.

Proposition 3.3. If $\left[\left(g_{1}, h_{1}\right)\right],\left[\left(g_{2}, h_{2}\right)\right]$ are mapped to the same element of the modular group via (3.7), then $h_{\mathbb{R}}\left(g_{1}\right)$ and $h_{\mathbb{R}}\left(g_{2}\right)$ must coincide.

Proof. By symmetry, it suffices to argue that the topological entropy of the system $g_{1}=h_{1} \circ f \circ h_{1}^{-1} \uparrow_{\hat{\mathbb{R}}}$ cannot exceed that of the system $g_{2}=h_{2} \circ f \circ h_{2}^{-1} \uparrow_{\hat{\mathbb{R}}}$. But these systems are conjugate with $f \uparrow_{h_{1}^{-1}(\hat{\mathbb{R}})}$ and $f \uparrow_{h_{2}^{-1}(\hat{\mathbb{R}})}$ respectively (keep in mind that the quasi-circles $h_{1}^{-1}(\hat{\mathbb{R}}), h_{2}^{-1}(\hat{\mathbb{R}})$ are $f$-invariant as the rational maps $g_{1}=h_{1} \circ f \circ h_{1}^{-1}, g_{2}=h_{2} \circ f \circ h_{2}^{-1}$ are with real coefficients.). Therefore, we only need to show that $h_{\text {top }}\left(f \uparrow_{h_{1}^{-1}(\hat{\mathbb{R}})}\right) \leq h_{\text {top }}\left(f \uparrow_{h_{2}^{-1}(\hat{\mathbb{R}})}\right)$.

Notice that if the automorphisms

$$
u_{1}: z \mapsto \overline{h_{1}^{-1}\left(\overline{h_{1}(z)}\right)}, \quad u_{2}: z \mapsto \overline{h_{2}^{-1}\left(\overline{h_{2}(z)}\right)}
$$

corresponding to $\left(g_{1}, h_{1}\right)$ and $\left(g_{2}, h_{2}\right)$ determine same classes in $\operatorname{Mod}(f)$, they must differ by a third quasi-conformal automorphism $v$ of $f$ isotopic to the identity: $u_{2}=u_{1} \circ v$. Thus there is an isotopy $\left\{v_{t}\right\}_{t \in[0,1]}$ from $v_{0}=v$ to $v_{1}=\mathbf{1}$ through q.c.-automorphisms of $f$. Thus $\left\{v_{t}\left(h_{1}^{-1}(\hat{\mathbb{R}})\right)\right\}_{t \in[0,1]}$ is a 1-parameter family of $f$ invariant quasi-circles terminating at $t=1$ with $h_{1}^{-1}(\hat{\mathbb{R}})$. Given a periodic point $z_{0}$ of $f,\left\{v_{t}\left(z_{0}\right)\right\}_{t \in[0,1]}$ is a curve of periodic points of $f$ since $v_{t}$ 's commute with $f$. 
The set of periodic points of $f$ is countable; therefore, all of these points coincide with $v_{1}\left(z_{0}\right)=x$. Consequently, $v_{0}=v=u_{1}^{-1} \circ u_{2}$ fixes each periodic point of $f$ and thus restricts to identity on the Julia set of $f$ since $\mathcal{J}(f)$ is the closure of repelling periodic points. Recalling the definitions of $u_{1}, u_{2}$ in (3.8), this indicates $h_{1}^{-1}\left(\overline{h_{1}(x)}\right)=h_{2}^{-1}\left(\overline{h_{2}(x)}\right)$ for any $x \in \mathcal{J}(f) \cap h_{1}^{-1}(\hat{\mathbb{R}})$. The left-hand side is just $x$ and hence $h_{2}(x)$ should be real; thus $\mathcal{J}(f) \cap h_{1}^{-1}(\hat{\mathbb{R}})$ is a closed subsystem of $f \uparrow_{h_{2}^{-1}(\hat{\mathbb{R}})}$ too. Now:

$$
h_{\text {top }}\left(f\left\lceil_{h_{1}^{-1}(\hat{\mathbb{R}})}\right)=h_{\text {top }}\left(f\left\lceil_{\mathcal{J}(f) \cap h_{1}^{-1}(\hat{\mathbb{R}})}\right) \leq h_{\text {top }}\left(f \uparrow_{h_{2}^{-1}(\hat{\mathbb{R}})}\right) .\right.\right.
$$

The same idea of Schwarz reflection appearing above can be employed to establish the $\mathcal{J}$-stable case as well.

Proof of Proposition 1.2. One characterization of $\mathcal{J}$-stability is the existence of a holomorphic motion of Julia sets [MnSS83, Theorem B]. Consequently, there is a continuous map

$$
i:[0,1] \times \mathcal{J}\left(f_{0}\right) \rightarrow \hat{\mathbb{C}}
$$

such that each $i_{t}:=i(t,$.$) is a conjugacy$

$$
\left(\mathcal{J}\left(f_{0}\right), f_{0}\left\lceil\mathcal{J}\left(f_{0}\right)\right) \rightarrow\left(\mathcal{J}\left(f_{t}\right), f_{t}\left\lceil\mathcal{J}\left(f_{t}\right)\right)\right.\right.
$$

with $i_{0}$ being identity. As every $f_{t}$ is with real coefficients, the Julia sets $\mathcal{J}\left(f_{t}\right)$ are invariant under the complex conjugation; and therefore, for any $x_{0} \in \mathcal{J}\left(f_{0}\right)$ the following is a well defined continuous map:

$$
t \in[0,1] \mapsto \overline{i_{t}^{-1}\left(\overline{i_{t}\left(x_{0}\right)}\right)} .
$$

The maps above commute with $f_{0}$ since $f_{t} \circ i_{t}=i_{t} \circ f_{0}$ and both $f_{0}$ and $f_{t}$ commute with complex conjugation. Hence if $x_{0}$ is picked to be periodic, (3.9) parametrizes a curve passing through the periodic points of $f_{0}$. In such a situation, (3.9) must be constant of value

$$
\overline{i_{0}^{-1}\left(\overline{i_{0}\left(x_{0}\right)}\right)}=x_{0}
$$

or equivalently $\overline{i_{t}\left(x_{0}\right)}=i_{t}\left(\overline{x_{0}}\right)$ for any $t \in[0,1]$. But $\mathcal{J}\left(f_{0}\right)$ contains all repelling periodic points of $f_{0}$ as a dense subset. So by continuity, $\overline{i_{t}(x)}=i_{t}(\bar{x})$ holds for all $x \in \mathcal{J}\left(f_{0}\right), t \in[0,1]$. In other words, the motion (3.11) of Julia sets respects the complex conjugation; in particular, it preserves the real points. The conjugacy (3.10) thus restricts to a conjugacy

$$
\left(\mathcal{J}\left(f_{0}\right) \cap \hat{\mathbb{R}}, f_{0}\left\lceil_{\mathcal{J}\left(f_{0}\right) \cap \hat{\mathbb{R}}}\right) \rightarrow\left(\mathcal{J}\left(f_{t}\right) \cap \hat{\mathbb{R}}, f_{t} \uparrow_{\mathcal{J}\left(f_{t}\right) \cap \hat{\mathbb{R}}}\right)\right.
$$

between two dynamical systems of topological entropies $h_{\mathbb{R}}\left(f_{0}\right)$ and $h_{\mathbb{R}}\left(f_{t}\right)$.

This discussion culminates in the following theorem:

Theorem 3.4. Given a real rational map $f \in \mathbb{R}(z)$ of degree $d \geq 2$, the real entropy function $h_{\mathbb{R}}: \mathcal{M}_{d}^{\prime}-\mathcal{S}^{\prime} \rightarrow[0, \log (d)]$ is constant on connected components of $\mathcal{M}_{d}^{\prime} \bigcap \mathrm{M}(f)-\mathcal{S}^{\prime}$.

\footnotetext{
${ }^{2}$ For any closed $f$-invariant subset $A$ of the Riemann sphere, the topological entropy of $f \Gamma_{A}$ coincides with that of the subsystem $f\lceil\mathcal{J}(f) \cap A$ Mil06a Problem 19-a].
} 
Proof. Proposition 3.3 says that the lift $\widetilde{h_{\mathbb{R}}}$ of $h_{\mathbb{R}}$ is constant on level sets of the continuous map $\{[(g, h)] \in \mathrm{T}(f) \mid g \in \mathbb{R}(z)$, Aut $(g)=\{\mathbf{1}\}\} \rightarrow \operatorname{Mod}(f)$ defined in (3.7). Since the target space is discrete, we conclude that $\widetilde{h_{\mathbb{R}}}:[(g, h)] \mapsto h_{\mathbb{R}}(g)$ is locally constant on the preceding domain. Now the commutative diagram (3.4) implies that $h_{\mathbb{R}}$ is locally constant on the image $\mathcal{M}_{d}^{\prime} \cap \mathrm{M}(f)-\mathcal{S}^{\prime}$ of this space in the moduli space $\mathcal{M}_{d}(\mathbb{C})$ and this finishes the proof.

Proof of Theorem 1.1. An immediate corollary of Proposition 2.7 and Theorem 3.4.

Of course, Theorem 3.4 is not interesting unless the intersection $\mathcal{M}_{d}^{\prime} \cap \mathrm{M}(f)$ is of positive dimension or equivalently, there are plenty of classes in the Teichmüller space $\mathrm{T}(f)$ which are represented by real maps.

Corollary 3.5. For a non-antipodal rational map $f \in \mathbb{R}(z)$ of degree $d \geq 2$ with $\operatorname{Aut}(f)=\{\mathbf{1}\}$, the real entropy function $h_{\mathbb{R}}: \mathcal{M}_{d}^{\prime}-\mathcal{S}^{\prime} \rightarrow[0, \log (d)]$ is constant on a submanifold of real dimension $\operatorname{dim}_{\mathbb{C}} \mathrm{M}(f)$ passing through $\langle f\rangle$.

Proof. The involution from Definition 3.1 acts on the $\mathrm{T}(f)$ via

$$
\iota:[(g, h)] \mapsto[(\tilde{g}, \tilde{h})] .
$$

Keep in mind that by identities (3.2), if $h$ is a q.c.-homeomorphisms of dilatation $\mu$ then $\tilde{h}$ would be another such homeomorphism of dilatation $\tilde{\mu}$. Hence the transformation $\iota$ from (3.12) acts on both $\mathrm{T}(f)$ and $\{[(g, h)] \in \mathrm{T}(f) \mid \operatorname{Aut}(g)=\{\mathbf{1}\}\}$ in a well defined manner because $\tilde{f}=f$ and the involution in (3.1) respects the composition and preserves the group of Möbius transformations. If $\operatorname{Aut}(g)=\{\mathbf{1}\}$ and $(g, h) \sim(\bar{g}, \tilde{h})$, then the maps $g$ and $\tilde{g}=\bar{g}$ are Möbius conjugate. Recalling Proposition 2.9, this implies that $g$ is with real coefficients if it is away from the antipodal locus. Nonetheless, the conditions of being on the antipodal locus or having non-trivial symmetries are closed. So if one can compute the dimension of Fix $(\iota: \mathrm{T}(f) \rightarrow \mathrm{T}(f))$ around a point $[(g, h)]=[(\tilde{g}, \tilde{h})]$ fixed by $\iota$ where $g \in \mathbb{R}(z)$ is non-antipodal and $\operatorname{Aut}(g)=\{\mathbf{1}\}$, then something can be inferred about the dimension $\mathcal{M}_{d}^{\prime} \cap \mathrm{M}(f)$.

To do so, suppose $f \in \mathbb{R}(z)$ is neither antipodal nor admits non-trivial Möbius symmetries. Clearly $[(f, \mathbf{1})]$ is a fixed point of the $C^{\infty}$ involution $\iota$ of the complex manifold $\mathrm{T}(f)$. The tangent map $\mathrm{d}_{[(f, \mathbf{1})]} \iota$ of $\iota$ at this point is a real-linear involution of the complex vector space $\mathrm{T}_{[(f, \mathbf{1})]}(\mathrm{T}(f))$. Thus the tangent space decomposes to the direct sum of +1 and -1 eigenspaces and the former is the tangent space to Fix $(\iota: \mathrm{T}(f) \rightarrow \mathrm{T}(f))$ at $[(f, \mathbf{1})]$. We claim the dimension of these two eigenspaces coincide. Any representative $(g, h)$ of a point in the Teichmüller space $\mathrm{T}(f)$ determines an $f$-invariant Beltrami differential $\mu(z) \frac{\mathrm{d} \bar{z}}{\mathrm{~d} z}$ where $\mu$ belongs to the unit ball in $L^{\infty}(\hat{\mathbb{C}})$. So there is a $\mathbb{C}$-linear surjection from the space

$$
\left\{\mu \in L^{\infty}(\hat{\mathbb{C}}) \mid \mu(z) \frac{\mathrm{d} \bar{z}}{\mathrm{~d} z} \text { is } f \text {-invariant. }\right\}
$$

onto the tangent space $\mathrm{T}_{[(f, \mathbf{1})]}(\mathrm{T}(f))$. Given the way $\iota$ is defined in (3.12) and remembering that for a q.c.-homeomorphism $h$ of dilatation $\mu$, the map $\tilde{h}$ is of dilatation $\tilde{\mu}$, we deduce that the involution $\mathrm{d}_{[(f, \mathbf{1})]} \iota$ of $\mathrm{T}_{[(f, \mathbf{1})]}(\mathrm{T}(f))$ comes from the involution $\mu \mapsto \tilde{\mu}$ acting on $L^{\infty}(\hat{\mathbb{C}})$. The description of this involution in (3.1) clearly indicates that this map is conjugate-linear and hence, so is $\mathrm{d}_{[(f, \mathbf{1})]} \iota$. This 
means scaler multiplication by i maps the eigenspace of +1 to that of -1 and vice versa. So, as real vector spaces, they are of dimension

$$
\operatorname{dim}_{\mathbb{C}} \mathrm{T}_{[(f, \mathbf{1})]}(\mathrm{T}(f))=\operatorname{dim}_{\mathbb{C}} \mathrm{T}(f)=\operatorname{dim}_{\mathbb{C}} \mathrm{M}(f) .
$$

The complex dimension of the Teichmüller space $\mathrm{T}(f)$, or equivalently that of the moduli space $\mathrm{M}(f)$, can be determined in terms of certain invariants of the holomorphic system $f: \hat{\mathbb{C}} \rightarrow \hat{\mathbb{C}}([$ MS98, Theorem 6.8], [Ahl06, p. 128]). Corollary 3.5 thus establishes a lower bound for the real dimension of the isentrope passing through a generic real rational map $f \in \mathbb{R}(z)$ in terms of the complex dynamics of $f$.

\section{EXAmples of Stable FAmilies of CONSTANT REAL ENTROPY}

This final section is devoted to examples of stable families of rational maps with real coefficients where, in accord with Theorem 1.1] and Proposition 1.2, the real entropy remains constant. We first treat the case of hyperbolic maps in 4.1 by a different approach utilizing the well known techniques of kneading theory [MT88. In $\$ 4.2$ we calculate the real entropy for the prominent family of flexible Lattès maps. At last, 4.3 entails a detailed discussion on families where the real entropy is the maximum $\log (d)$.

4.1. Hyperbolic components. Recall that a rational map is called hyperbolic if each of its critical orbits converges to an attracting periodic cycle Mil06a, Theorem 19.1]. Such maps form an open subset of the moduli space $\mathcal{M}_{d}(\mathbb{C})$ whose connected components are called hyperbolic components. Hyperbolic maps are known to be $\mathcal{J}$-stable and thus, Proposition 1.2 implies that each connected component of the intersection with $\mathcal{M}_{d}^{\prime}$ of a hyperbolic component in $\mathcal{M}_{d}(\mathbb{C})$ is included in a single isentrope. Here, we present a completely different proof of this fact that also sheds light on the entropy values realized by real hyperbolic maps.

Theorem 4.1. Let $d \geq 3$ and $\mathcal{U}$ be a connected component of the intersection of a hyperbolic component of $\mathcal{M}_{d}(\mathbb{C})$ with the real subvariety $\mathcal{M}_{d}^{\prime}$. Then the function $h_{\mathbb{R}}$ is constant over $\mathcal{U}$ with a value which is the logarithm of an algebraic number.

Remark 4.2. There is a thorough classification of hyperbolic components of $\mathcal{M}_{2}(\mathbb{C})$ Mil93, Ree90. In the case of $d=2$ Theorem 4.1 still remains valid but requires a little bit of more work as excluding the symmetry locus might cause the real hyperbolic component $\mathcal{U}$ to become disconnected; cf. [Fil19.

Proof of Theorem 4.1. The open subset $\mathcal{U}-\mathcal{S}^{\prime}$ of $\mathcal{M}_{d}^{\prime}$ is connected because $\mathcal{U} \cap \mathcal{S}^{\prime}$ is of codimension at least two; see Proposition 2.4. Any real representative $f \in \mathbb{R}(z)$ of a point in it restricts to a continuous multimodal circle map $f \uparrow_{\hat{\mathbb{R}}}: \hat{\mathbb{R}} \rightarrow \hat{\mathbb{R}}$ that satisfies the hypothesis of Lemma 4.3 below. Therefore, $\log \left(h_{\mathbb{R}}(\langle f\rangle)\right)$ is always algebraic for a point $\langle f\rangle$ from this open connected set. The continuity of $h_{\mathbb{R}}$ : $\mathcal{M}_{d}^{\prime}-\mathcal{S}^{\prime} \rightarrow[0, \log (d)]$ then yields its constancy over $\mathcal{U}-\mathcal{S}^{\prime}$

Lemma 4.3. Let $I$ be an interval $[a, b]$ or a circle. Let $f: I \rightarrow I$ be a continuous multimodal self-map of I whose turning points are attracted by periodic orbits. Then the number $\mathrm{e}^{h_{\mathrm{top}}(f)}$ is algebraic. 
Proof. Let us first deal with the interval case. We need to use the classical kneading theory of Milnor and Thurston developed in [MT88. Suppose $f:[a, b] \rightarrow[a, b]$ is a multimodal self-map of $I:=[a, b]$ with turning points $c_{1}<\cdots<c_{l-1}$ and laps $I_{1}=\left[a, c_{1}\right], I_{2}=\left[c_{1}, c_{2}\right], \ldots, I_{l-1}=\left[c_{l-2}, c_{l-1}\right], I_{l}=\left[c_{l-1}, b\right]$. The shape of the restriction $f \Gamma_{n_{n}}$ of $f$ to the $n^{\text {th }}$ lap $I_{n}$ will be denoted by $\epsilon_{n} \in\{ \pm 1\}$ which is +1 if the restriction is increasing and -1 when it is decreasing. To each point $x \in I$, one can assign infinite vectors $\left(\theta_{i}\left(x^{+}\right)\right)_{i \geq 0}$ and $\left(\theta_{i}\left(x^{-}\right)\right)_{i \geq 0}$ whose components come from the set of symbols $\left\{ \pm I_{1}, \ldots, \pm I_{l}\right\}$. Here is the definition: $\theta_{i}\left(x^{+}\right)=\epsilon I_{n}$ (respectively $\left.\theta_{i}\left(x^{-}\right)=\epsilon I_{n}\right)$ means that there is a half-open interval with its left end (resp. right end) at $x$ on which $f^{\circ i}$ is monotonic of shape $\epsilon \in\{ \pm 1\}$ and is mapped by $f^{\circ i}$ into $I_{n}$. Associated with them are formal power series $\theta\left(x^{+}\right):=$ $\sum_{i=0}^{\infty} \theta_{i}\left(x^{+}\right) t^{i}$ and $\theta\left(x^{-}\right):=\sum_{i=0}^{\infty} \theta_{i}\left(x^{-}\right) t^{i}$ from $V[[t]]$ with $V$ being the free abelian group $\mathbb{Z} .\left\{I_{1}, \ldots, I_{l}\right\}$. The next definition is that of the kneading increments of $f$ given by $\nu_{m}:=\theta\left(c_{m}^{+}\right)-\theta\left(c_{m}^{-}\right)$for any $1 \leq m \leq l-1$. One can then form the $(l-1) \times l$ kneading matrix $N$ of power series in $\mathbb{Z}[[t]]$ with $N_{m n}$ being the coefficient of $I_{n}$ in $\nu_{m}$, i.e. $\nu_{m}=\sum_{n=1}^{l} N_{m n} I_{n}$. Denoting the orientation of $f \uparrow_{I_{n}}$ by $\epsilon_{n} \in\{ \pm 1\}$ and the determinant of the submatrix of $N$ obtained from deleting the $n^{\text {th }}$ column by $D_{n}(t)$, it can be proved that the formal power series $(-1)^{n+1} D_{n}(t) /\left(1-\epsilon_{n} t\right)$ is independent of $1 \leq n \leq l$. This common power series is called the kneading invariant of $f$ and will be denoted by $D(t)$. It is easy to observe that the coefficients of $D(t)$ are bounded integers, so $D(t)$ defines an analytic function on the disk $|t|<1$. Here is the main result ( MT88, Theorem 6.3]):

If $h_{\text {top }}(f)=0, D(t)$ does not have any root in the open unit disk and otherwise, $\mathrm{e}^{-h_{\mathrm{top}}(f)}$ is the smallest root of $D(t)$ in $[0,1)$.

Thus it suffices to show that under our assumption the kneading invariant $D(t)$ is a rational map with integer coefficients whose roots, including $\mathrm{e}^{-h_{\text {top }}(f)}$, are algebraic numbers. In order to do so, one just needs to show that kneading coordinates $\left(\theta_{i}\left(c_{m}^{+}\right)\right)_{i \geq 0}$ and $\left(\theta_{i}\left(c_{m}^{-}\right)\right)_{i>0}$ are eventually periodic. We will argue that $\left(\theta_{i}\left(c_{m}^{+}\right)\right)_{i \geq 0}$ is periodic; the case of $\left(\theta_{i}\left(c_{m}^{-}\right)\right)_{i \geq 0}$ is completely similar. Suppose for $i \geq q, f^{\circ i}\left(c_{m}\right)$ is in the immediate basin of the periodic point $x_{i \bmod p}$ from the orbit $x_{0} \mapsto x_{1} \mapsto$ $\cdots \mapsto x_{p-1} \mapsto x_{0}$. We claim that for $\delta>0$ small enough, one can take $q^{\prime}>q$ so large that for $i>q^{\prime}$ the interval $f^{\circ i}\left(\left(c_{m}, c_{m}+\delta\right)\right)$ is in the interior of a lap $I_{n_{i \bmod p}}$ of $f$ which is dependent only on the remainder of $i$ modulo $p$. To see this, fix $0 \leq r<p$. Note that $f^{\circ(j p+r)}\left(c_{m}\right) \rightarrow x_{r}$ as $j \rightarrow \infty$; and so if $x_{r}$ is not a turning point, $f^{\circ(j p+r)}\left(c_{m}\right)$ and hence the image under $f^{\circ(j p+r)}$ of small enough non-degenerate subintervals $\left[c_{m}, c_{m}+\delta\right]$ of the basin belong to the interior of the lap that has $x_{r}$ for large enough $j$ 's; say for $j \geq j_{r}$. The same holds even when $x_{r}$ is a turning point of $f$; we only have to rule out the possibility of $f^{\circ(j p+r)}\left(c_{m}\right)$ alternating between the two laps that have $x_{r}$ in common: if the fixed point

$$
x_{r}=\lim _{j \rightarrow \infty} f^{\circ(j p+r)}\left(\left(c_{m}, c_{m}+\delta\right)\right)
$$

of $f^{\circ p}$ is a turning point as well, then for $j$ large enough, $f^{\circ(j p+r)}\left(\left(c_{m}, c_{m}+\delta\right)\right)$ always lands to the left of $x_{r}$ if $x_{r}$ is a local maximum of $f^{\circ p}$ and to the right if it is a local minimum. Repeating this argument for all $r \in\{0, \ldots, p-1\}$, $p \cdot\left(\max \left\{j_{0}, \ldots, j_{p-1}\right\}+1\right)$ then works as the desired $q^{\prime}$.

Next, after decreasing $\delta$ if necessary, suppose all iterates $f, \ldots, f^{\circ\left(q^{\prime}+1\right)}$ restrict to monotonic maps on $\left[c_{m}, c_{m}+\delta\right]$. We now show that $\left(\theta_{i}\left(c_{m}^{+}\right)\right)_{i \geq 0}$ is periodic of period 
$2 p$ for $i>q^{\prime}: f^{\circ i}\left(\left(c_{m}, c_{m}+\delta\right)\right)$ is contained in the lap $I_{n_{i \bmod p}}$, and the degree of $f^{\circ i} \uparrow_{\left[c_{m}, c_{m}+\delta\right]}$ is the product of the degree of the monotonic map $f^{\circ}\left(q^{\prime}+1\right) \uparrow_{\left[c_{m}, c_{m}+\delta\right]}$ by the degrees of $f$ on laps $I_{n_{\left(q^{\prime}+1\right) \bmod p}}, \ldots, I_{n_{(i-1) \bmod p}}$. Replacing $i$ with $i+2 p$ does not affect the former while multiplies the latter product by $\left(\prod_{i=1}^{p} \epsilon_{n_{i \bmod p}}\right)^{2}=1$.

Finally, notice that kneading theory has also been developed for continuous multimodal circle maps or equivalently, for multimodal interval maps with finitely many discontinuities; see [Pre89, appendix]. Therefore, Lemma 4.3 is valid for multimodal circle map too.

Remark 4.4. Much more can be said about arithmetic properties of entropy values of post-critically finite multimodal maps. The paper [Thu14] establishes that a real algebraic integer arises as $\exp \left(h_{\text {top }}(f: I \rightarrow I)\right)$ for a critically finite multimodal map $f: I \rightarrow I$ if and only if it is a weak Perron number, i.e. at least as large as the absolute values of its Galois conjugates.

4.2. The Lattès family. A family of flexible Lattès maps of the same degree is an example of a quasi-conformally trivial family of non-hyperbolic maps. (A detailed treatment of Lattès maps can be found in [Mil06b].) Conjecturally, these are the only families of rational maps admitting invariant line fields. Any such family forms a single dynamical moduli space of complex dimension one. The example below verifies Theorem 3.4 through calculating the real entropy of those flexible Lattès maps that preserve the real circle. It is well known that the Julia set of a Lattès map is the whole Riemann sphere and hence, unlike 4.1 the subsystem obtained from restricting to the real circle can never have an attractor.

Example 4.5. Set $d=m^{2} \geq 4$ for an integer $m \neq 0, \pm 1$ and consider a flexible Lattès map $f$ of degree $d$ obtained from the multiplication by $m$ map $[m]:[z] \mapsto$ $[m z]$ on the elliptic curve $E=\mathbb{C} / \mathbb{Z}+\mathbb{Z} \tau$ with $\tau$ being in the upper half plane. So $f$ makes the following diagram commutative where the columns are the twofold ramified covering $\pi: E \rightarrow \mathbb{P}^{1}(\mathbb{C})$ obtained by taking the quotient of $E$ by the action of the involution $[z] \mapsto[-z]$; the morphism which is induced by the Weierstrass function $\wp: \mathbb{C} \rightarrow \widehat{\mathbb{C}}$ of the lattice $\Lambda:=\mathbb{Z}+\mathbb{Z} \tau$.

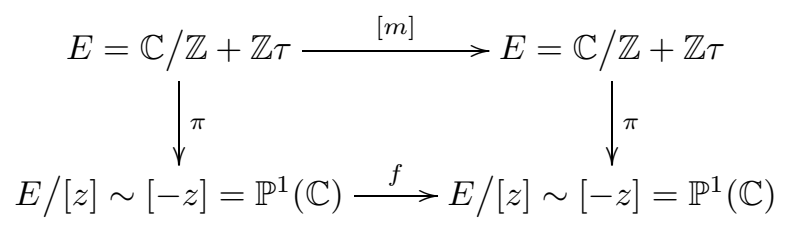

The map $f$ preserves $\hat{\mathbb{R}}$ if the Weierstrass function

$$
\wp(z)=\frac{1}{z^{2}}+\sum_{0 \neq w \in \mathbb{Z}+\mathbb{Z} \tau}\left(\frac{1}{(z-w)^{2}}-\frac{1}{w^{2}}\right)
$$

commutes with the complex conjugation; for instance, when the lattice $\mathbb{Z}+\mathbb{Z} \tau$ is invariant under the complex conjugation. This happens if and only if $\operatorname{Re}(\tau) \in \frac{1}{2} \mathbb{Z}$. Up to the action of $\mathrm{SL}_{2}(\mathbb{Z}), \operatorname{Re}(\tau)$ can then be assumed to be 0 or $\frac{1}{2}$. Let us work with the former and in the case of $\operatorname{Re}(\tau)=\frac{1}{2}$ one merely needs to replace $\tau$ with $\tau-\frac{1}{2}$ in the subsequent discussion.

Assuming that the period $\tau$ is purely imaginary, let us investigate the induced map $f$ and the corresponding dynamics on the real circle. We shall do so by pulling 
back to the dynamics on $\wp^{-1}(\hat{\mathbb{R}})$ or on the invariant subset $\pi^{-1}(\hat{\mathbb{R}})$ of the elliptic curve $E$. Since $\wp(\bar{z})=\overline{\wp(z)}, \wp(z)$ is real if and only if either $2 \operatorname{Re}(z)$ or $2 \operatorname{Im}(z)$ i belongs to the rectangular lattice $\Lambda=\mathbb{Z}+\mathbb{Z} \tau$. We conclude that $\wp^{-1}(\hat{\mathbb{R}})$ is the countable set of lines parallel to axes in the complex plane whose $x$ and $y$ intercepts come from $\frac{1}{2} \mathbb{Z}$ and $\frac{\operatorname{Im}(\tau)}{2} \mathbb{Z}$. This collection determines a tessellation of the complex plane with the smaller rectangle of vertices $0, \frac{1}{2}, \frac{\tau+1}{2}$ and $\frac{\tau}{2}$ (in the counterclockwise order) which is bijectively mapped onto $\hat{\mathbb{R}}$ via $\wp$.

The union $\wp^{-1}(\hat{\mathbb{R}})$ of lines in $\mathbb{C}$ projects onto two pairs of parallel circle on the torus $E=\mathbb{C} / \mathbb{Z}+\mathbb{Z} \tau$

$$
\left(\{[x] \mid x \in \mathbb{R}\},\left\{\left[x+\frac{\tau}{2}\right] \mid x \in \mathbb{R}\right\}\right), \quad\left(\{[x \tau] \mid x \in \mathbb{R}\},\left\{\left[x \tau+\frac{1}{2}\right] \mid x \in \mathbb{R}\right\}\right)
$$

that intersect each other in four points (the 2-torsion points) and constitute $\pi^{-1}(\hat{\mathbb{R}})$. So the topological entropy of $f \uparrow_{\hat{\mathbb{R}}}$ coincides with that of the restriction of $[\mathrm{m}]$ to this union of circles because $\pi$ establishes a finite degree semi-conjugacy between these two systems. According to the parity of $m$, on each circle from the preimage $\pi^{-1}(\hat{\mathbb{R}})$ the map $[m]$ restricts to the multiplication by $m$ onto another circle from this union. We conclude that $h_{\mathbb{R}}(f)$ is the topological entropy of

$$
S^{1}=\mathbb{R} / \mathbb{Z} \rightarrow S^{1}=\mathbb{R} / \mathbb{Z}:[x] \mapsto[m x],
$$

i.e. $\log (|m|)$. Notice that under $\pi$ each circle form (4.2) is mapped onto a compact subinterval of $\hat{\mathbb{R}}$; and if the circle is preserved by the multiplication map $[m]$, the dynamics on the interval is given by the quotient of (4.3) by the involution $[x] \mapsto$ $[-x]$, i.e. a Chebyshev polynomial. The dynamics on $\hat{\mathbb{R}}$ is illustrated in Figure 1
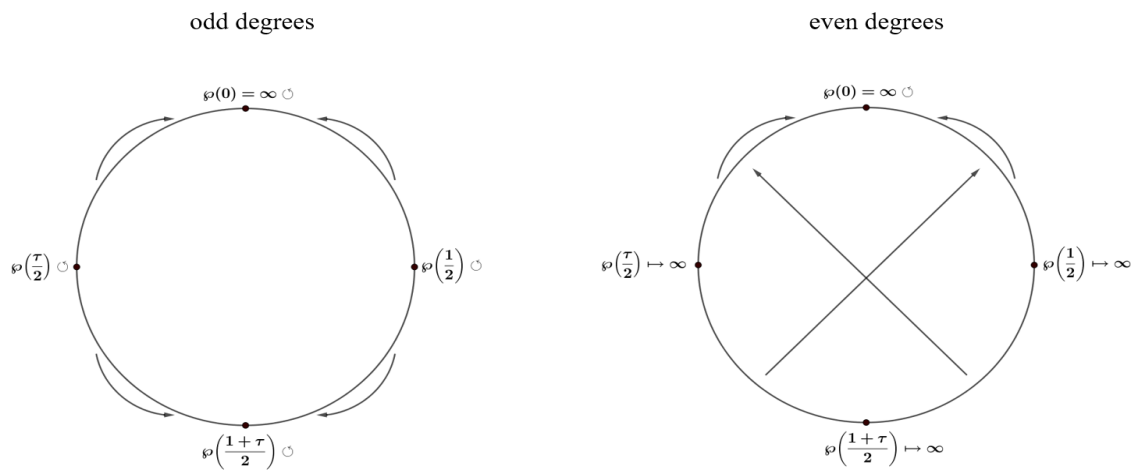

Figure 1. The real dynamics of the map $f$ from (4.1) in odd and even degrees. The images under $\pi$ of circles (4.2) on the elliptic curve cut $\hat{\mathbb{R}}$ into four intervals determined by the values of the Weierstrass function at 2-torsion points. Under $f$, each interval is either preserved or is mapped onto another interval. The dynamics on invariant intervals is that of a Chebyshev polynomial. 
Example 4.6. Let us calculate the real entropy of a couple of rigid (not flexible) Lattès maps. It is known that any Lattès map semi-conjugate to an endomorphism of an elliptic curve $E$ via a morphism $E \rightarrow \mathbb{P}^{1}(\mathbb{C})$ whose degree (unlike the case of the diagram (4.1) ) is greater than two must originate from elliptic curves with extra automorphisms, i.e. the elliptic curves corresponding to square and hexagonal lattices Mil06b.

In the case of the square elliptic curve, the rational map $f(z):=-\frac{1}{4}\left(z+\frac{1}{z}-2\right)$ fits into the commutative diagram below.

$$
\begin{aligned}
& \left\{y^{2}=x^{3}-x\right\}=\mathbb{C} / \mathbb{Z}+\mathbb{Z} \mathrm{i} \longrightarrow\left\{y^{2}=x^{3}-x\right\}=\mathbb{C} / \mathbb{Z}+\mathbb{Z} \mathrm{i}
\end{aligned}
$$

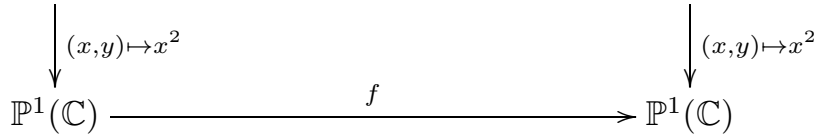

The second iterate of $f$ would be a finite quotient of the endomorphism $[1+\mathrm{i}]^{\circ 2}=$ [2i]. But in the Legendre form $y^{2}=x^{3}-x$, the automorphism defined via the multiplication by $\mathrm{i}$ is just $(x, y) \mapsto(-x, \pm \mathrm{i} y)$; so $f^{\circ 2}$ is in fact induced via the multiplication by two endomorphism and hence, based on the discussion in Example 4.5. we deduce that:

$$
h_{\mathbb{R}}(f)=\frac{1}{2} h_{\mathbb{R}}\left(f^{\circ 2}\right)=\frac{1}{2} \log (2)=\log (\sqrt{2}) .
$$

We finish this example with the entropy calculation for a real map which is the quotient of an endomorphism of the hexagonal elliptic curve $\mathbb{C} / \mathbb{Z}+\mathbb{Z} \omega$ where $\omega:=\mathrm{e}^{\frac{2 \pi \mathrm{i}}{3}}$ is a primitive third root of unity. Consider the commutative diagram below.

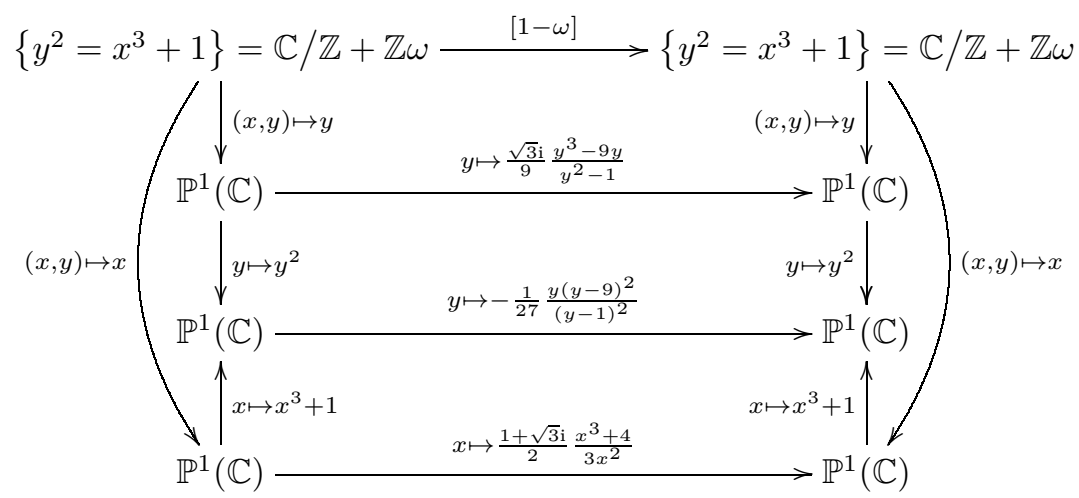

Only the map appeared in the third row, i.e. $f(z):=-\frac{1}{27} \frac{z(z-9)^{2}}{(z-1)^{2}}$, is real. The map $x \mapsto x^{3}+1$ from the diagram above establishes a semi-conjugacy of finite degree between the flexible Lattès map induced by multiplication by $[-3]$ and $f^{\circ 2}$. The real entropy of the former has been calculated in Example 4.5 as $\log (3)$. We conclude that:

$$
h_{\mathbb{R}}(f)=\frac{1}{2} h_{\mathbb{R}}\left(f^{\circ 2}\right)=\frac{1}{2} \log (3)=\log (\sqrt{3}) .
$$

4.3. Maps of maximal real entropy. The isentrope $h_{\mathbb{R}}=\log (d)$ is an interesting one to investigate. In this subsection we carry out a thorough analysis of this isentrope that culminates in the proof of Theorem 1.3 
Theorem 4.7. Let $f \in \mathbb{R}(z)$ be of degree $d \geq 2$ with $\mu_{f}$ its measure of maximal entropy. Then the following are equivalent:

(a) $\mu_{f}(\hat{\mathbb{R}})>0$;

(b) the Julia set $\mathcal{J}(f)$ is a subset of $\hat{\mathbb{R}}$;

(c) $h_{\text {top }}\left(f \uparrow_{\hat{\mathbb{R}}}: \hat{\mathbb{R}} \rightarrow \hat{\mathbb{R}}\right)=\log (d)$.

Proof. It is clear that $(\mathrm{b}) \Rightarrow(\mathrm{a}),(\mathrm{c})$; because if $\mathcal{J}(f) \subseteq \hat{\mathbb{R}}$, using $\operatorname{supp}\left(\mu_{f}\right)=\mathcal{J}(f)$ :

$$
\begin{aligned}
h_{\text {top }}\left(f \uparrow_{\hat{\mathbb{R}}}\right) & \geq h_{\text {top }}\left(f\left\lceil_{\mathcal{J}(f)}\right)\right. \\
& \geq h_{\mu_{f}}\left(f\lceil\mathcal{J}(f))=h_{\mu_{f}}(f: \hat{\mathbb{C}} \rightarrow \hat{\mathbb{C}})=h_{\text {top }}(f: \hat{\mathbb{C}} \rightarrow \hat{\mathbb{C}})=\log (d) .\right.
\end{aligned}
$$

Next, suppose $\mu_{f}(\hat{\mathbb{R}})>0$. According to Montel's theorem, for any open neighborhood $U \subseteq \hat{\mathbb{C}}$ of a point of $\mathcal{J}(f), \bigcup_{n} f^{-\circ n}(U)$ includes a dense open subset of $\mathcal{J}(f)$. We use the properties of the measure of maximal entropy to show that this union is of full measure with respect to $\mu_{f}$. It is known that $\mu_{f}$ is ergodic and satisfies $f^{*} \mu_{f}=d . \mu_{f}$ [FLMn83. The union $\bigcup_{n} f^{-o n}(U)$ is backward-invariant, so must be of zero measure if it is not of full measure. But this measure zero set is an open subset of the Julia set; thus there is an iterate of $f$ that maps it onto the set $\mathcal{J}(f)$ with full measure [Mil06a, Corollary 14.2]. This contradicts $f^{*} \mu_{f}=d . \mu_{f}$; we deduce that $\mu_{f}\left(\bigcup_{n} f^{-o n}(U)\right)=1$. Now fixing a countable collection of open sets $\left\{U_{m}\right\}_{m}$ for which $\left\{U_{m} \cap \mathcal{J}(f)\right\}_{m}$ is an open basis for the topology of $\mathcal{J}(f)$ and then taking the intersection over $U_{m}$ 's, we arrive at the full measure subset $\bigcap_{m} \bigcup_{n} f^{-\circ n}\left(U_{m}\right)$ which by the Baire category theorem contains a dense subset of $\mathcal{J}(f)$. Furthermore, the forward iterates of any of its points form a dense subset of $\mathcal{J}(f)$. As $\mu_{f}(\hat{\mathbb{R}})>0$, this full measure subset intersects $\hat{\mathbb{R}}$ : There is a point on $\hat{\mathbb{R}}$ whose orbit is dense in $\mathcal{J}(f)$. But $\hat{\mathbb{R}}$ is closed and forward-invariant so must contain the whole $\mathcal{J}(f)$.

At last, we show (c) $\Rightarrow($ b) that will yields (c) $\Rightarrow(a)$ because we have already established $(\mathrm{a}) \Leftrightarrow(\mathrm{b})$. To this end, we invoke the main result of the article Hof81. indicating that a multimodal transformation of an interval with positive entropy admits a measure of maximal entropy. In that reference, multimodal maps can be discontinuous at turning points. Thus, identifying $\hat{\mathbb{R}} \cong S^{1}$ with $[0,1)$ via the bijection $x \mapsto \mathrm{e}^{2 \pi \mathrm{i} x}$, the same holds for continuous circle maps of positive entropy. So there is a Borel probability measure, say $\nu$, on the circle $\hat{\mathbb{R}}$ with respect to which the metric entropy of $f \uparrow_{\hat{\mathbb{R}}}: \hat{\mathbb{R}} \rightarrow \hat{\mathbb{R}}$ coincides with its topological entropy $\log (d)$. Pushing forward via the inclusion $i: \hat{\mathbb{R}} \hookrightarrow \hat{\mathbb{C}}$, one gets a measure $i_{*} \nu$ on the Riemann sphere with respect to which the measure theoretic entropy of $f: \hat{\mathbb{C}} \rightarrow \hat{\mathbb{C}}$ is at least $\log (d)$. So $i_{*} \nu$ is a measure of maximal entropy for this rational map too. The uniqueness of the measure of maximal entropy for rational maps established in [Mn83] then implies that $i_{*} \nu=\mu_{f}$. Hence $\mu_{f}(\hat{\mathbb{R}})=\nu(\hat{\mathbb{R}})=1$.

Now that we know $\mathcal{J}(f)$ must be completely real once $h_{\mathbb{R}}(f)=\log (d)$, we will elaborate on the dynamics on the Julia set below; compare with [EvS11, Theorem 2].

Theorem 4.8. Let $f \in \mathbb{R}(z)$ be a rational map of degree $d \geq 2$ with $h_{\mathbb{R}}(f)=$ $\log (d)$. Then precisely one of the followings holds:

(a) There is a real fixed point $p$ of multiplier $\lambda \in[-1,1]$ whose immediate basin of attraction $\mathcal{A}$ is the only Fatou component; and a compact interval 
$I \subset \hat{\mathbb{R}}$ with $f^{-1}(I) \subsetneq I$ whose complement $\hat{\mathbb{R}}-I$ is the largest open interval included in $\mathcal{A}$. In this case, the Julia set is a Cantor set included in $I$.

(b) The same as above but with $f^{-1}(I)=I$. In this case, $\mathcal{J}(f)$ coincides with the interval I restricted to which $f \uparrow_{I}: I \rightarrow I$ is a boundary-anchored $(d-1)$-modal map with surjective laps. The only Fatou component is the $\operatorname{basin} \mathcal{A}=\hat{\mathbb{C}}-I$.

(c) The Fatou components are the open half planes and are immediate basins either for a pair of conjugate non-real attracting periodic points, or for a parabolic real fixed point which is either of multiplier -1 or of multiplier +1 and multiplicity 3 . In this case, the Julia set is $\hat{\mathbb{R}}$ and $f \uparrow_{\hat{\mathbb{R}}}: \hat{\mathbb{R}} \rightarrow \hat{\mathbb{R}}$ is a d-sheeted unramified covering.

Proof. By Theorem 4.7 the Julia set $\mathcal{J}(f)$ is contained in $\hat{\mathbb{R}}$. Recall that the Julia set is always either connected or has uncountably many connected components [Mil06a, Corollary 4.15]. So $\mathcal{J}(f) \subseteq \hat{\mathbb{R}}$ is either a non-degenerate subinterval of $\hat{\mathbb{R}}$, the whole circle or a disjoint union of (forcibly at most countably many) compact non-degenerate subintervals of the real circle with a (necessarily uncountable) closed totally disconnected subset of it. We claim that in the latter case there is not any subinterval and thus $\mathcal{J}(f)$ is a closed totally disconnected subset of $\hat{\mathbb{R}}$ and hence (given the fact that it has no isolated point) a Cantor set. Assume the contrary; $\mathcal{J}(f)$ admits both interval and singleton components. But $f$ takes a connected component of $\mathcal{J}(f)$ to another such component and, being a non-constant analytic map, cannot collapse a non-degenerate interval to a point. So the forward iterates of an interval component never cover a singleton component. This is a contradiction since the former contains an open subset of $\hat{\mathbb{R}}$ and thus an open subset of $\mathcal{J}(f)$, and by Montel's theorem the union of forward images of any non-empty open subset of the Julia set is the whole Julia set. Consequently, $\mathcal{J}(f) \subseteq \hat{\mathbb{R}}$ is either $\hat{\mathbb{R}}$, a subinterval of it or a Cantor set on it.

Since $\mathcal{J}(f) \subseteq \hat{\mathbb{R}}$, there are at most two Fatou components with equality if and only if $\mathcal{J}(f)$ coincides with $\hat{\mathbb{R}}$. Each Fatou component is periodic and cannot be a rotation domain due to the fact that there are only finitely many Fatou component. Hence, invoking the classification of Fatou components, each Fatou components of $f$ is the immediate basin of an attracting or parabolic periodic point $p$. If $p$ lies on $\hat{\mathbb{R}}$, its multiplier $\lambda$ belongs to $[-1,1]$. If $p$ is not real, its complex conjugate $\bar{p}$ determines a different Fatou component of the real map $f$ and so the Fatou components appear in a conjugate pair; hence $\mathcal{J}(f)=\hat{\mathbb{R}}$ and we are in the situation that (c) describes. Notice that $p, \bar{p}$ cannot be parabolic as they do not belong to $\mathcal{J}(f)=\hat{\mathbb{R}}$. For $p$ real, the only other case where $\mathcal{J}(f)$ coincides with $\hat{\mathbb{R}}$ is if $p$ is a parabolic point with the half planes as its immediate basins. The period of $p$ must be one: the Fatou components appear in conjugate pairs and as there are merely two of them, there cannot be a parabolic cycle of period larger than one. Having two (or equivalently more than one) parabolic basins of attraction is immediate when $\lambda=-1$ ([Mil06a, Lemma 10.4]), while requires the multiplicity of the fixed point to be 3 when $\lambda=1$.

In parts (a) and (b) the Julia set is a proper closed subset of $\hat{\mathbb{R}}$ and hence there is a single Fatou component which is the basin of attraction $\mathcal{A}$ for a fixed point $p \in \hat{\mathbb{R}}$ of multiplier $\lambda \in[-1,1]$. The connected component of $\mathcal{A} \cap \hat{\mathbb{R}}$ around $p$ is a forward-invariant open interval which is the immediate basin of the fixed 
point $p$ of the real system $f \uparrow_{\hat{\mathbb{R}}}: \hat{\mathbb{R}} \rightarrow \hat{\mathbb{R}}$. Thus its complement, denoted by $I$, is a compact subinterval of $\hat{\mathbb{R}}$ containing $\mathcal{J}(f)$ and satisfying $f^{-1}(I) \cap \hat{\mathbb{R}} \subseteq I$. We have the equality in (b), where $f^{-1}(I)=I$, while the inclusion is strict in (a). In the latter case where $f^{-1}(I) \cap \hat{\mathbb{R}} \subsetneq I$, the proper subset $\mathcal{J}(f)$ of $I$ must be Cantor as it cannot be any smaller subinterval of $I$ because then, the connected component around $p$ of the intersection of the Fatou set with $\hat{\mathbb{R}}$ would be larger than $\hat{\mathbb{R}}-I$ contradicting the definition of $I$. We claim that the preimage of $I$ is totally real again and hence $f^{-1}(I) \subsetneq I$. Assume otherwise. The backward-invariant Cantor subset $\mathcal{J}(f)$ of $I$ is totally real and hence there must be a subinterval of the complement $I-\mathcal{J}(f) \subset \hat{\mathbb{R}}$ whose preimage is not completely real. This can be written as $\left(y_{0}, y_{0}+\epsilon_{0}\right)$ or $\left(y_{0}-\epsilon_{0}, y_{0}\right)$ where $\epsilon_{0}>0$ and $y_{0} \in \mathcal{J}(f)$. There is no loss of generality in going with the former and also taking $\epsilon_{0}>0$ so small that there is no critical value in $\left(y_{0}, y_{0}+\epsilon_{0}\right)$. The preimage of $\left(y_{0}, y_{0}+\epsilon_{0}\right)$ under the degree $d$ rational map $f: \hat{\mathbb{C}} \rightarrow \widehat{\mathbb{C}}$ then consists of $d$ disjoint curves on the Riemann sphere each homeomorphic to the open interval $(0,1)$ and either contained in $\hat{\mathbb{R}}$ or completely away from it. The non-real ones among them must appear in conjugate pairs as $f$ is with real coefficients. But the closures of members of any such pair must have a point of $f^{-1}\left(y_{0}\right) \subset \mathcal{J}(f) \subset \hat{\mathbb{R}}$ in common, namely a real Julia point $x_{0}$. We deduce that $f$ is not locally injective at $x_{0}$; in particular, $y_{0}$ is a critical value of $f$. Switching to the interval $\left(y_{0}-\epsilon_{1}, y_{0}+\epsilon_{1}\right)$ symmetric with respect to $y_{0}$ with $0<\epsilon_{1}<\epsilon_{0}$ so small that this interval has no other critical value other than $y_{0}$, the component $S$ of $f^{-1}\left(\left(y_{0}-\epsilon_{1}, y_{0}+\epsilon_{1}\right)\right)$ around $x_{0} \in f^{-1}\left(y_{0}\right)$ is a "starshaped" subset of the plane symmetric with respect to the real axis with "arms" homeomorphic to open intervals that are mapped onto $\left(y_{0}-\epsilon_{1}, y_{0}+\epsilon_{1}\right)$ via $f$ and, aside from the one which is a real open subinterval containing $x_{0}$, the rest are curves that intersect $\hat{\mathbb{R}}$ only at $x_{0}$. Such "non-real arms" of $S \subseteq f^{-1}\left(\left(y_{0}-\epsilon_{1}, y_{0}+\epsilon_{1}\right)\right)$ exist since $x_{0}$ is a critical point of $f$. But $y_{0} \in \mathcal{J}(f) \subset \hat{\mathbb{R}}$ cannot be an isolated point of $\mathcal{J}(f)$, so every arm of the star $S$ has a Julia point distinct from $x_{0}$. Therefore, there are non-real Julia points; a contradiction.

To finish the proof, we need to address the extra information that parts (b) and (c) provide for cases where $\mathcal{J}(f)$ is "smooth", namely is an interval or the whole circle. Since the Julia set is backward-invariant, $f\lceil\mathcal{J}(f): \mathcal{J}(f) \rightarrow \mathcal{J}(f)$ is a degree $d$ ramified covering whose ramification structure may be readily investigated. First, suppose $\mathcal{J}(f)=\hat{\mathbb{R}}$. If there is a critical point of $f$ on $\hat{\mathbb{R}}$, then

$$
\hat{\mathbb{R}}-f^{-1}(\{v \in \hat{\mathbb{R}} \mid v \text { a critical value of } f: \hat{\mathbb{C}} \rightarrow \hat{\mathbb{C}}\})
$$

is a disjoint union of

$$
\# f^{-1}(\{v \in \hat{\mathbb{R}} \mid v \text { a critical value of } f: \hat{\mathbb{C}} \rightarrow \hat{\mathbb{C}}\})
$$

open intervals restricted to which $f$ yields a covering map onto

$$
\hat{\mathbb{R}}-\{v \in \hat{\mathbb{R}} \mid v \text { a critical value of } f: \hat{\mathbb{C}} \rightarrow \hat{\mathbb{C}}\} ;
$$

a union of

$$
\#\{v \in \hat{\mathbb{R}} \mid v \text { a critical value of } f: \hat{\mathbb{C}} \rightarrow \hat{\mathbb{C}}\}
$$

intervals. So $f$ takes each of the former intervals bijectively onto one of the latter. But $\mathcal{J}(f)=\hat{\mathbb{R}}$ is backward-invariant under the degree $d$ map $f$, so each of the 
latter intervals has to be covered by $d$ of the former ones. This happens only when

$$
\begin{aligned}
& \# f^{-1}(\{v \in \hat{\mathbb{R}} \mid v \text { a critical value of } f: \hat{\mathbb{C}} \rightarrow \hat{\mathbb{C}}\}) \\
& \quad=d(\#\{v \in \hat{\mathbb{R}} \mid v \text { a critical value of } f: \hat{\mathbb{C}} \rightarrow \widehat{\mathbb{C}}\}) ;
\end{aligned}
$$

that is, when $f$ admits $d$ points over each critical value lying on $\hat{\mathbb{R}}$, which is absurd and therefore, $f \uparrow_{\mathcal{J}(f)}: \mathcal{J}(f) \rightarrow \mathcal{J}(f)$ is a degree $d$ (unramified) covering of circles. Finally, when $\mathcal{J}(f)$ is an interval, $f\lceil\mathcal{J}(f): \mathcal{J}(f) \rightarrow \mathcal{J}(f)$ is a multimodal interval map of topological entropy $\log (d)$. By (1.8) this entropy cannot be realized if the lap number is less than $d$. Therefore, $f\lceil\mathcal{J}(f)$ is $(d-1)$-modal. On each of its $d$ laps $f\lceil\mathcal{J}(f)$ restricts to a surjective map onto $\mathcal{J}(f)$ because otherwise, there would be a non-empty open subinterval of $\mathcal{J}(f)$ over points of which $f\lceil\mathcal{J}(f)$ admits less than $d$ preimages. The same must hold for fibers of $f: \hat{\mathbb{C}} \rightarrow \hat{\mathbb{C}}$ above these points due to the backward-invariance of $\mathcal{J}(f)$ contradicting $\operatorname{deg} f=d$. The map has to be boundary-anchored too: if $f$ takes an endpoint of the interval $\mathcal{J}(f)$ to a point of its interior, by continuity, it maps points outside the interval sufficiently close to that endpoint inside $\mathcal{J}(f)$; a contradiction since the points outside the interval are Fatou points.

Proof of Theorem 1.3. As proved in Theorem 4.7 $h_{\mathbb{R}}(f)=\log (d)$ implies $\mathcal{J}(f) \subseteq$ $\hat{\mathbb{R}}$. The detailed discussion in the subsequent Theorem 4.8 showed that $\mathcal{J}(f)$ must be either a Cantor set on the real circle, a subinterval of it or the entirety of $\hat{\mathbb{R}}$, as outlined in parts (a), (b) and (c) of Theorem 4.8 respectively. Replacing $f \in \mathbb{R}(z)$ with its conjugate

$$
z \mapsto\left(z \mapsto \frac{z-\mathrm{i}}{z+\mathrm{i}}\right) \circ f \circ\left(z \mapsto \frac{z-\mathrm{i}}{z+\mathrm{i}}\right)^{-1},
$$

it is no loss of generality to assume that the degree $d$ rational map $f$ preserves the unit circle $|z|=1$ instead with its Julia set a subset of the circle. This is in particular the case when $f$ restricts to a degree $\pm d$ self-cover of the unit circle since then the closed backward-invariant set $|z|=1$ must contain $\mathcal{J}(f)$ by Montel's theorem. Such a map $f$ can be described by a Blaschke product

$$
\mathrm{e}^{2 \pi \mathrm{i} c} \prod_{i=1}^{d}\left(\frac{z-a_{i}}{1-\overline{a_{i} z}}\right) \quad\left(\left|a_{1}\right|, \ldots,\left|a_{d}\right|<1 ; c \in \mathbb{R} / \mathbb{Z}\right)
$$

as in (1.2) or its post-composition with $z \mapsto \frac{1}{z}$, based on whether the degree of the induced circle map is $+d$ or $-d$. There is not any critical point on the unit circle and hence, $\mathcal{J}(f)$ cannot be a subinterval of the circle because Theorem 4.8(b) indicates that in such a situation, $\mathcal{J}(f)$ contains critical points. Also notice that any degree $d$ map with the whole unit circle as its Julia set is one of these Blaschke products due to the fact that in such a situation $f\left\{_{\{|z|=1\}}:\{|z|=1\} \rightarrow\{|z|=1\}\right.$ must be a $d$-sheeted covering according to Theorem 4.8(c). We can next address parts (i) and (ii) of Theorem 1.3, the map $f$ given by $\mathrm{e}^{2 \pi \mathrm{ic}} \prod_{i=1}^{d}\left(\frac{z-a_{i}}{1-\overline{a_{i} z}}\right)$ (or its post-composition with $z \mapsto \frac{1}{z}$ ) is hyperbolic if and only if it either has an attracting fixed point on $|z|=1$ - in which case $\mathcal{J}(f)$ would be a Cantor subset of $|z|=1$ - or has a fixed point (necessarily attracting by Schwartz Lemma) inside the open unit disk - in which case $\mathcal{J}(f)$ coincides with the unit circle. These are the possibilities appeared respectively in (a) and (c) of Theorem 4.8 with the real circle in place of 
the unit circle. Equation (1.4) means that the point $\mathrm{e}^{2 \pi \mathrm{i} \theta_{0}}$ on the unit circle is fixed by $f(z)=\mathrm{e}^{2 \pi \mathrm{i} c} \prod_{i=1}^{d}\left(\frac{z-a_{i}}{1-\bar{a}_{i} z}\right)$ and (1.3) guarantees that it is attracting: a simple logarithmic differentiation shows that on the unit circle (cf. [Mar83, Proposition 1])

$$
\frac{f^{\prime}(z)}{f(z)}=\frac{1}{z} \sum_{i=1}^{d} \frac{1-\left|a_{i}\right|^{2}}{\left|z-a_{i}\right|^{2}}
$$

and the multiplier of $\mathrm{e}^{2 \pi \mathrm{i} \theta_{0}}$ is thus of magnitude $\sum_{i=1}^{d} \frac{1-\left|a_{i}\right|^{2}}{\left|\mathrm{e}^{2 \pi \mathrm{i} \theta_{0}}-a_{i}\right|^{2}}$. As for the part (ii) of Theorem 1.3 where $f$ possesses a fixed point in the open unit disk, one can put the fixed point at the origin via conjugation with a suitable biholomorphism of the open unit disk. Therefore, there is a conjugacy that preserves the unit circle and transforms $f(z)$ to another Blaschke product of the form

$$
\mathrm{e}^{2 \pi \mathrm{i} c^{\prime}} z \cdot \prod_{i=1}^{d-1}\left(\frac{z-a_{i}^{\prime}}{1-\bar{a}_{i}^{\prime} z}\right) .
$$

One can kill the only degree of freedom left by conjugating with a rotation of the unit circle and getting rid of $\mathrm{e}^{2 \pi \mathrm{i} c^{\prime}}$. This leaves us with a Blaschke product of the form

$$
z . \prod_{i=1}^{d-1}\left(\frac{z-a_{i}^{\prime \prime}}{1-\overline{a_{i}^{\prime \prime} z}}\right) .
$$

(or its composition with $z \mapsto \frac{1}{z}$ that has $0 \mapsto \infty \mapsto 0$ as an attracting 2-cycle).

Part (iii) of Theorem 1.3 is straightforward. (We have formulated part (iii) for the original real circle as calculating the multiplicity of a parabolic point of the Blaschke product (1.2) on the unit circle leads to complicated algebraic expressions.) Invoking Theorem 4.8(c), the degree $d$ map $f \in \mathbb{R}(z)$ here must have non-real critical points and a parabolic fixed point of multiplier $\epsilon= \pm 1$ on $\hat{\mathbb{R}}$ whose multiplicity should be 3 if $\epsilon=+1$. Without any loss of generality, we may assume that the fixed point is $\infty$. Therefore, $f$ can be written as $f(z)=\epsilon\left(z+\frac{P(z)}{Q(z)}\right)$ with $P, Q$ coprime polynomials satisfying $\operatorname{deg} P \leq d-1$ and $\operatorname{deg} Q=d-1$. For $\epsilon=1$ we moreover want the multiplicity of the fixed point $z=\infty$ of $f(z)=z+\frac{P(z)}{Q(z)}$ to be more than 2 and it is not hard to see that this is equivalent to $\operatorname{deg} P<d-1$.

Finally, we turn to part (iv) of Theorem 1.3. Given a degree $d$ map $f$ with $\mathcal{J}(f)$ a subinterval of $\hat{\mathbb{R}}$, we claim that there is a lift of $f$ via a two-sheeted branched covering of $\pi: \hat{\mathbb{C}} \rightarrow \hat{\mathbb{C}}$ to another rational map $g$ whose Julia set is an analytic circle on the Riemann sphere. This will be proved separately in Proposition 4.9. After suitable changes of coordinates in the domain and the co-domain of $\pi$, we may assume that $\mathcal{J}(g)$ is either the unit circle or the real circle and the degree two map $\mathcal{J}(g) \rightarrow \mathcal{J}(f)$ induced by the semi-conjugacy $\pi$ is either

$$
\left\{\begin{array}{l}
\{|z|=1\} \rightarrow[-2,2] \\
z \mapsto z+\frac{1}{z}
\end{array} ;\right.
$$

or

$$
\left\{\begin{array}{l}
\hat{\mathbb{R}} \rightarrow[0,+\infty] \\
z \mapsto z^{2}
\end{array} .\right.
$$


The map $g$ then fits in the description provided by parts (ii) and (iii) of Theorem 1.3. In the latter case (the non-hyperbolic situation) $g$ is in the form of $\epsilon\left(z+\frac{P(z)}{Q(z)}\right)$ with $\hat{\mathbb{R}}$ as its Julia set and the semi-conjugacy is the map $z \mapsto z^{2}$ from (4.5). Hence $f$ is the quotient of $\epsilon\left(z+\frac{P(z)}{Q(z)}\right)$ by the action of $z \mapsto-z$. One can easily verify that this Möbius transformation commutes with $\epsilon\left(z+\frac{P(z)}{Q(z)}\right)$ if and only if one of the polynomials $P(z), Q(z)$ is even and the other is odd. In the hyperbolic case, the semi-conjugacy is the Joukowsky map $z \mapsto z+\frac{1}{z}$ from (4.4) and thus $f$ must be the quotient of a Blaschke product of the form (1.2) that has the unit circle as its Julia set by the action of $z \mapsto \frac{1}{z}$. Following previous discussions regarding the part (ii) of Theorem 1.3 $g$ must have an attracting fixed point inside the unit disk which can assumed to be zero; and hence is in the form of

$$
\mathrm{e}^{2 \pi \mathrm{i} c} z \cdot \prod_{i=1}^{d-1}\left(\frac{z-a_{i}}{1-\bar{a}_{i} z}\right)
$$

It is not hard to check that the map above commutes with $z \mapsto \frac{1}{z}$ if and only if $\mathrm{e}^{2 \pi \mathrm{ic}}= \pm 1$ and the points $a_{1}, \ldots, a_{d-1}$ of the open unit disk are located symmetrically with respect to the real axis; hence (1.6).

Next, we have the proposition below that concludes the proof of Theorem 1.3 and furthermore, provides a description of rational maps with interval Julia sets.

Proposition 4.9. Given a rational map $f$ of degree $d$ whose Julia set is the interval $[-2,2]$, there exists a rational map $g$ of degree $d$ with the unit circle as its Julia set which is semi-conjugate to $f$

$$
\mathfrak{j} \circ g=f \circ \mathfrak{j}
$$

via the Joukowsky map

$$
\mathfrak{j}(z):=z+\frac{1}{z} .
$$

Proof. We denote the restrictions of (4.7) to upper and lower unit semi-circles by $\mathfrak{j}_{+}$and $\mathbf{j}_{-}$.

$$
\left\{\begin{array}{l}
\mathfrak{j}_{+}:\left\{e^{2 \pi \mathrm{i} \theta} \mid 0 \leq \theta \leq \pi\right\} \rightarrow[-2,2] \\
e^{2 \pi \mathrm{i} \theta} \mapsto 2 \cos (\theta)
\end{array} \quad ; \quad\left\{\begin{array}{l}
\mathfrak{j}_{-}:\left\{e^{2 \pi \mathrm{i} \theta} \mid \pi \leq \theta \leq 2 \pi\right\} \rightarrow[-2,2] \\
e^{2 \pi \mathrm{i} \theta} \mapsto 2 \cos (\theta)
\end{array} .\right.\right.
$$

These diffeomorphisms are respectively orientation-reversing and orientationpreserving once the unit semi-circles and the interval $[-2,2]$ are equipped with orientations inherited from the obvious positive orientations of the unit circle and the real line. Theorem 4.8(b) indicates that $f\left\lceil_{[-2,2]}:[-2,2] \rightarrow[-2,2]\right.$ has $d$ surjective monotonic pieces. Hence indexing its turning points as

$$
t_{0}=-2<t_{1}<\cdots<t_{d-1}<t_{d}=2,
$$

the map $f$ takes each subinterval $\left[t_{i}, t_{i+1}\right]$ bijectively onto $[-2,2]$ with these bijections alternatingly increasing and decreasing. Consider the preimages of points in (4.9) under the maps in (4.8):

$$
p_{i+}=\mathfrak{j}_{+}^{-1}\left(t_{i}\right) \quad p_{i-}=\mathfrak{j}_{-}^{-1}\left(t_{i}\right) \quad(0<i<d) .
$$


Clearly, one has

(4.11) $\mathfrak{j}_{ \pm}^{-1}(2)=1<p_{(d-1)+}<\cdots<p_{1+}<\mathfrak{j}_{ \pm}^{-1}(-2)=-1<p_{1-}<\cdots<p_{(d-1)-}$;

in the counter-clockwise direction of the unit circle. Moving along the unit circle from 1 to -1 and then back to 1 , the points above cut $2 d$ consecutive closed $\operatorname{arcs} C_{1}, \ldots, C_{2 d}$ with the first $d$ covering the closed upper semi-circle and the rest covering the closed lower semi-circle. These arcs are disjoint aside from the adjacent arcs $C_{i}$ and $C_{i+1}$ (indices considered cyclically modulo $2 d$ ) intersecting at a point from (4.11). Next, we define a self-map $g$ of the unit circle as follows:

$$
g\left\lceil C_{i}=\left\{\begin{array}{ll}
\left(\mathfrak{j}_{+}\right)^{-1} \circ f \circ \mathfrak{j}_{+}\left\lceil_{C_{i}}\right. & \text { if } 1 \leq i \leq d \& i \text { odd } \\
\left(\mathfrak{j}_{-}\right)^{-1} \circ f \circ \mathfrak{j}_{+} \Upsilon_{C_{i}} & \text { if } 1 \leq i \leq d \& i \text { even } \\
\left(\mathfrak{j}_{+}\right)^{-1} \circ f \circ \mathfrak{j}_{-} \Upsilon_{C_{i}} & \text { if } d+1 \leq i \leq 2 d \& i \text { odd } \\
\left(\mathfrak{j}_{-}\right)^{-1} \circ f \circ \mathfrak{j}_{-} \Upsilon_{C_{i}} & \text { if } d+1 \leq i \leq 2 d \& i \text { even }
\end{array} \quad(1 \leq i \leq 2 d) .\right.\right.
$$

The map is clearly well defined since adjacent arcs overlap at points whose image under $\mathfrak{j}$ (and so under $\mathfrak{j}_{+}$or $\mathfrak{j}_{-}$, as appropriate) is a point from $f^{-1}(\{ \pm 2\})$ in (4.9); and we have $\mathfrak{j}_{ \pm}^{-1}(2)=\{1\}$ and $\mathfrak{j}_{ \pm}^{-1}(-2)=\{-1\}$. It is evident from (4.12) that $g$ bijects each of the arcs $C_{1}, \ldots, C_{2 d}$ alternatingly onto either the upper or the lower semi-circle. Notice that these diffeomorphisms are of the same degree; which is, 1 if the last lap

$$
f\left\lceil\left[t_{d-1}, t_{d}\right]=\mathbf{j}_{+}\left(C_{1}\right)=\mathfrak{j}_{-}\left(C_{2 d}\right)\right.
$$

is increasing and -1 if the lap is decreasing. For $1 \leq i<d$ (respectively or $d+1 \leq i<2 d)$, the diffeomorphisms $\left(\mathfrak{j}_{+}\right)^{-1} \circ f \circ \mathfrak{j}_{+}\left\lceil C_{i}\right.$ and $\left(\mathfrak{j}_{-}\right)^{-1} \circ f \circ \mathfrak{j}_{+}\left\lceil C_{i+1}\right.$ (resp. $\left(\mathbf{j}_{+}\right)^{-1} \circ f \circ \mathbf{j}_{-} \Gamma_{C_{i}}$ and $\left.\left(\mathbf{j}_{-}\right)^{-1} \circ f \circ \mathbf{j}_{-} \Gamma_{C_{i+1}}\right)$ are of the same degree as the degrees of $\mathfrak{j}_{ \pm}$and also those of the adjacent laps $f \uparrow_{\mathfrak{j}\left(C_{i}\right)}, f \uparrow_{\mathfrak{j}\left(C_{i+1}\right)}$ are opposite. The same is true for $i=d$, when it comes to the degrees of $g\left\lceil C_{d}=\mathfrak{j}_{(-1)^{d-1}}^{-1} \circ f \circ \mathfrak{j}_{+}\left\lceil C_{d}\right.\right.$ and $g\left\lceil C_{d+1}=\mathfrak{j}_{(-1)^{d}}^{-1} \circ f \circ \mathfrak{j}_{-}\left\lceil_{C_{d+1}}\right.\right.$ : they are just $(-1)^{d-1}$ times the degree of the lap

$$
f \uparrow_{\mathfrak{j}_{+}}\left(C_{d}\right)=\mathfrak{j}_{-}\left(C_{d+1}\right)=\left[t_{0}, t_{1}\right] .
$$

The discussion above implies that (4.12) defines an unramified $d$-sheeted selfcover $g$ of the unit circle satisfying (4.6). The map is clearly real analytic away from the endpoints of $C_{i}$ 's (as appeared in (4.10) ) where the formula for $g$ changes. Thus every interior point $x$ on an arc $C_{i}$ admits an open neighborhood $U_{x}$ in the complex plane on which there is an extension of $g$ to a local biholomorphism. This holomorphic extension of $g$ of course satisfies $\mathfrak{j} \circ g=f \circ \mathfrak{j}$ as well. Now notice that the unit circle is the preimage of the Julia set $[-2,2]$ of $f$ under $\mathfrak{j}$. By the complete invariance of the Julia set, any such extension of $g$ takes points off the unit circle to points off the unit circle. As this holomorphic extension preserves the orientation, it must take points out of the unit disk inside and vice versa if $g\left\lceil C_{i}\right.$ is orientation-reversing; while it keeps $U_{x} \cap\{|z|<1\}$ and $U_{x} \cap\{|z|>1\}$ invariant if $g\left\lceil_{C_{i}}\right.$ is orientation-preserving. We conclude that there is a holomorphic extension of the circle map $g$ to the open neighborhood $U:=\bigcup_{x} U_{x}$ of the complement

$$
\{|z|=1\}-\left\{p_{1 \pm}, \ldots, p_{(d-1) \pm}, \pm 1\right\}
$$

of the endpoints (4.11) of arcs $C_{1}, \ldots, C_{2 d}$; and this extension, denoted by the same symbol $g$, either preserves or interchanges $U \cap\{|z|<1\}$ and $U \cap\{|z|>1\}$, based on whether the degree of the unramified covering

$$
g\lceil\{|z|=1\}:\{|z|=1\} \rightarrow\{|z|=1\}
$$


is positive or negative respectively. But either on the open unit disk or on its complement the map $\mathbf{j}$ from (4.7) restricts to a biholomorphism onto the Fatou set $\hat{\mathbb{C}}-[-2,2]$ of $f$. Consequently, away from the unit circle one can solve $\mathfrak{j} \circ g=f \circ \mathfrak{j}$ for $g$ : if the degree of the circle map (4.13) is $d, g\left\lceil_{U \cap\{|z|<1\}}\right.$ and $g\left\lceil_{U \cap\{|z|>1\}}\right.$ are given by

$$
\left(\mathfrak { j } \lceil _ { \{ | z | < 1 \} } : \{ | z | < 1 \} \rightarrow \hat { \mathbb { C } } - [ - 2 , 2 ] ) ^ { - 1 } \circ f \circ \left(\mathfrak{j}\left\lceil_{\{|z|<1\}}:\{|z|<1\} \rightarrow \hat{\mathbb{C}}-[-2,2]\right)\right.\right.
$$

and

$$
\left(\mathfrak{j}\left\lceil_{\{|z|>1\}}:\{|z|>1\} \rightarrow \hat{\mathbb{C}}-[-2,2]\right)^{-1} \circ f \circ(\mathfrak{j}\lceil\{|z|>1\}:\{|z|>1\} \rightarrow \hat{\mathbb{C}}-[-2,2]) ;\right.
$$

while they are respectively given by

$$
\left(\mathfrak { j } \lceil _ { \{ | z | > 1 \} } : \{ | z | > 1 \} \rightarrow \hat { \mathbb { C } } - [ - 2 , 2 ] ) ^ { - 1 } \circ f \circ \left(\mathfrak{j}\left\lceil_{\{|z|<1\}}:\{|z|<1\} \rightarrow \hat{\mathbb{C}}-[-2,2]\right)\right.\right.
$$

and

$$
\left(\mathfrak{j}\left\lceil_{\{|z|<1\}}:\{|z|<1\} \rightarrow \hat{\mathbb{C}}-[-2,2]\right)^{-1} \circ f \circ(\mathfrak{j}\lceil\{|z|<1\}:\{|z|>1\} \rightarrow \hat{\mathbb{C}}-[-2,2]) ;\right.
$$

if the degree of (4.13) is $-d$. We conclude that $g$ can be holomorphically extended even further to $\hat{\mathbb{C}}-\left\{p_{1 \pm}, \ldots, p_{(d-1) \pm}, \pm 1\right\}$. By the Identity Principle, the extension again satisfies the functional equation $\mathfrak{j} \circ g=f \circ \mathfrak{j}$. Consequently, for any sequence $\left\{a_{n}\right\}_{n}$ tending to one of the points of the unit circle

$$
\left\{p_{1 \pm}, \ldots, p_{(d-1) \pm}, \pm 1\right\}=\mathfrak{j}^{-1}\left(f^{-1}(\{ \pm 2\})\right)
$$

excluded above, any convergent subsequence of $\left\{g\left(a_{n}\right)\right\}_{n}$ tends to an element of $\mathfrak{j}^{-1}(\{ \pm 2\})=\{ \pm 1\}$. We deduce that none of these isolated points is an essential singularity and so $g$ extends holomorphically to the whole Riemann sphere. Therefore, we have constructed a rational function $g \in \mathbb{C}(z)$ semi-conjugate to $f$ as in (4.6). Its Julia set is of course the preimage of $\mathcal{J}(f)=[-2,2]$ under the Joukowsky map $\mathfrak{j}$; namely, the unit the circle. This finishes the proof.

Remark 4.10. The dynamics on the Julia set can be easily described in many cases of Theorem 4.8 In 4.8(a), the Julia dynamics is conjugate to the one-sided shift on $d$ symbols if the critical points are away from the Julia set [Prz96]. In 4.8(b), the dynamics on the Julia set is that of a boundary-anchored expansive interval map with $d$ surjective laps, hence conjugate to a piecewise linear map of the same modality. Finally, in 4.8 (c) where $\mathcal{J}(f)=\hat{\mathbb{R}}$, once $f$ is without parabolic fixed points, $f \uparrow_{\hat{\mathbb{R}}}: \hat{\mathbb{R}} \rightarrow \hat{\mathbb{R}}$ would be an expanding circle map of degree $d$ and thus conjugate to one of the self-covers $z \mapsto z^{d}$ or $z \mapsto z^{-d}$ of the unit circle [Shu69]. In both 4.8 (b) and 4.8 (c), the multiplier of any periodic point of period $n$ of $f$ is $\pm d^{n}$ except the unique non-repelling fixed point; compare with [Mis81, Theorem 8.1].

Example 4.11. The examples of quadratic rational maps appeared in Mil06a, Problem 10-e] embody all possibilities for the Julia set outlined in Theorem 4.8 The following examples all possess a parabolic fixed point at infinity. For maps $\pm\left(z-\frac{1}{z}\right)$ the Julia set is $\hat{\mathbb{R}}$. If $f(z)=z+\frac{1}{z}-2$, the Julia set is the completely invariant interval $I=[0,+\infty]$. Finally, for $f(z)=z-\frac{1}{z}+1$ the Julia set is a Cantor subset of the interval $I=[-\infty, 1]$; and we have

$$
f^{-1}([-\infty, 1])=[-\infty,-1] \cup[0,1] \subsetneq[-\infty, 1] .
$$


Notice that the first two examples above (with circle and interval Julia sets) are semi-conjugate as predicted by Theorem [1.3. $\left(z-\frac{1}{z}\right)^{2}=z^{2}+\frac{1}{z^{2}}-2$. One other example of such semi-conjugacies is given by Chebyshev polynomials: the $d^{\text {th }}$ Chebyshev polynomial $T_{d}(z)$ (normalized to be monic via a linear conjugation) satisfies $T_{d}\left(z+\frac{1}{z}\right)=z^{d}+\frac{1}{z^{d}}$; its Julia set is $[-2,2]$, the image of the unit circle which is the Julia set of $z \mapsto z^{d}$ under the semi-conjugacy $z \mapsto z+\frac{1}{z}$. Compare with [Mil06a, Problems 7-c \& 7-d].

\section{REFERENCES}

[Ahl06] Lars V. Ahlfors, Lectures on quasiconformal mappings, 2nd ed., University Lecture Series, vol. 38, American Mathematical Society, Providence, RI, 2006. With supplemental chapters by C. J. Earle, I. Kra, M. Shishikura and J. H. Hubbard. MR2241787

[BBM15] Araceli Bonifant, Xavier Buff, and John Milnor, Antipode preserving cubic maps: the fjord theorem, Proc. Lond. Math. Soc. (3) 116 (2018), no. 3, 670-728, DOI 10.1112/plms.12075. MR3772619

[Bro76] Roger W. Brockett, Some geometric questions in the theory of linear systems, IEEE Trans. Automatic Control AC-21 (1976), no. 4, 449-455, DOI 10.1109/tac.1976.1101301. MR0469386

[BvS15] Henk Bruin and Sebastian van Strien, Monotonicity of entropy for real multimodal maps, J. Amer. Math. Soc. 28 (2015), no. 1, 1-61, DOI 10.1090/S0894-0347-201400795-5. MR.3264762

[DH85] A. Douady and J. H. Hubbard, Étude dynamique des polynômes complexes. Partie $I$ (French), Publications Mathématiques d'Orsay [Mathematical Publications of Orsay], vol. 84, Université de Paris-Sud, Département de Mathématiques, Orsay, 1984. MR762431

[Dou95] Adrien Douady, Topological entropy of unimodal maps: monotonicity for quadratic polynomials, Real and complex dynamical systems (Hillerød, 1993), NATO Adv. Sci. Inst. Ser. C Math. Phys. Sci., vol. 464, Kluwer Acad. Publ., Dordrecht, 1995, pp. 65-87. MR 1351519

[EvS11] Alexandre Eremenko and Sebastian van Strien, Rational maps with real multipliers, Trans. Amer. Math. Soc. 363 (2011), no. 12, 6453-6463, DOI 10.1090/S0002-99472011-05308-0. MR 2833563

[Fat19] P. Fatou, Sur les équations fonctionnelles (French), Bull. Soc. Math. France 47 (1919), 161-271. MR1504787

[Fil19] Khashayar Filom. Monotonicity of entropy for real quadratic rational maps. arXiv e-prints, Jan 2019.

[FLMn83] Alexandre Freire, Artur Lopes, and Ricardo Mañé, An invariant measure for rational maps, Bol. Soc. Brasil. Mat. 14 (1983), no. 1, 45-62, DOI 10.1007/BF02584744. MR736568

[Hof81] Franz Hofbauer, On intrinsic ergodicity of piecewise monotonic transformations with positive entropy. II, Israel J. Math. 38 (1981), no. 1-2, 107-115, DOI 10.1007/BF02761854. MR599481

[HQ15] Ruben A. Hidalgo and Saul Quispe, On Real and Pseudo-Real Rational Maps, arXiv e-prints, page arXiv:1502.05306. February 2015.

[Lyu82] M. Yu. Lyubich, The measure of maximal entropy of a rational endomorphism of a Riemann sphere (Russian), Funktsional. Anal. i Prilozhen. 16 (1982), no. 4, 78-79. MR684138

[Mar83] N. F. G. Martin, On finite Blaschke products whose restrictions to the unit circle are exact endomorphisms, Bull. London Math. Soc. 15 (1983), no. 4, 343-348, DOI 10.1112/blms/15.4.343. MR703758

[Mil93] John Milnor, Geometry and dynamics of quadratic rational maps, Experiment. Math. 2 (1993), no. 1, 37-83. With an appendix by the author and Lei Tan. MR.1246482

[Mil06a] John Milnor, Dynamics in one complex variable, 3rd ed., Annals of Mathematics Studies, vol. 160, Princeton University Press, Princeton, NJ, 2006. MR.2193309

[Mil06b] John Milnor, On Lattès maps, Dynamics on the Riemann sphere, Eur. Math. Soc., Zürich, 2006, pp. 9-43, DOI 10.4171/011-1/1. MR2348953 
[Mis81] Michał Misiurewicz, Absolutely continuous measures for certain maps of an interval, Inst. Hautes Études Sci. Publ. Math., (53):17-51, 1981.

[Mis95] Michał Misiurewicz, Continuity of entropy revisited, Dynamical systems and applications, World Sci. Ser. Appl. Anal., vol. 4, World Sci. Publ., River Edge, NJ, 1995, pp. 495-503, DOI 10.1142/9789812796417_0031. MR1372979

[Mn83] Ricardo Mañé, On the uniqueness of the maximizing measure for rational maps, Bol. Soc. Brasil. Mat. 14 (1983), no. 1, 27-43, DOI 10.1007/BF02584743. MR736567

[MnSS83] R. Mañé, P. Sad, and D. Sullivan, On the dynamics of rational maps, Ann. Sci. École Norm. Sup. (4) 16 (1983), no. 2, 193-217. MR732343

[MP77] Michał Misiurewicz and Feliks Przytycki, Topological entropy and degree of smooth mappings (English, with Russian summary), Bull. Acad. Polon. Sci. Sér. Sci. Math. Astronom. Phys. 25 (1977), no. 6, 573-574. MR 458501

[MS80] M. Misiurewicz and W. Szlenk, Entropy of piecewise monotone mappings, Studia Math. 67 (1980), no. 1, 45-63, DOI 10.4064/sm-67-1-45-63. MR579440

[MS98] Curtis T. McMullen and Dennis P. Sullivan, Quasiconformal homeomorphisms and dynamics. III. The Teichmüller space of a holomorphic dynamical system, Adv. Math. 135 (1998), no. 2, 351-395, DOI 10.1006/aima.1998.1726. MR1620850

[MSW17] Nikita Miasnikov, Brian Stout, and Phillip Williams, Automorphism loci for the moduli space of rational maps, Acta Arith. 180 (2017), no. 3, 267-296, DOI 10.4064/aa85486-2017. MR 3709645

[MT88] John Milnor and William Thurston, On iterated maps of the interval, Dynamical systems (College Park, MD, 1986), Lecture Notes in Math., vol. 1342, Springer, Berlin, 1988, pp. 465-563, DOI 10.1007/BFb0082847. MR970571

[MT00] John Milnor and Charles Tresser, On entropy and monotonicity for real cubic maps, Comm. Math. Phys. 209 (2000), no. 1, 123-178, DOI 10.1007/s002200050018. With an appendix by Adrien Douady and Pierrette Sentenac. MR.1736945

[Pre89] Chris Preston, What you need to know to knead, Adv. Math. 78 (1989), no. 2, 192-252, DOI 10.1016/0001-8708(89)90033-9. MR.1029100

[Prz96] Feliks Przytycki, Iterations of rational functions: which hyperbolic components contain polynomials?, Fund. Math. 149 (1996), no. 2, 95-118, DOI 10.4064/fm-149-2-95-118. MR 1376666

[Ree90] M. Rees, Components of degree two hyperbolic rational maps, Invent. Math. 100 (1990), no. 2, 357-382, DOI 10.1007/BF01231191. MR1047139

[Shu69] Michael Shub, Endomorphisms of compact differentiable manifolds, Amer. J. Math. 91 (1969), 175-199, DOI 10.2307/2373276. MR240824

[Sil98] Joseph H. Silverman, The space of rational maps on $\mathbf{P}^{1}$, Duke Math. J. 94 (1998), no. 1, 41-77, DOI 10.1215/S0012-7094-98-09404-2. MR.1635900

[Sil07] Joseph H. Silverman, The arithmetic of dynamical systems, Graduate Texts in Mathematics, vol. 241, Springer, New York, 2007. MR2316407

[SS85] Michael Shub and Dennis Sullivan, Expanding endomorphisms of the circle revisited, Ergodic Theory Dynam. Systems 5 (1985), no. 2, 285-289, DOI 10.1017/S014338570000290X. MR796755

[Thu14] William P. Thurston, Entropy in dimension one, Frontiers in complex dynamics, Princeton Math. Ser., vol. 51, Princeton Univ. Press, Princeton, NJ, 2014, pp. 339-384. MR.3289916

[Yoc02] Jean-Christophe Yoccoz, Analytic linearization of circle diffeomorphisms, Dynamical systems and small divisors (Cetraro, 1998), Lecture Notes in Math., vol. 1784, Springer, Berlin, 2002, pp. 125-173, DOI 10.1007/978-3-540-47928-4_3. MR1924912

Department of Mathematics, University of Michigan, 530 Church Street, Ann Arbor, MiCHigAN 48109

Email address: filom@umich.edu 